\title{
Effect of sawtooth oscillations on energetic ions*
}

\author{
Ya. I. Kolesnichenko ${ }^{a}$, V. V. Lutsenko ${ }^{a}$, R. B. White $^{b}$, Yu. V. Yakovenko ${ }^{a}$ \\ ${ }^{a}$ Scientific Centre "Institute for Nuclear Research", Kyiv, 03680, Ukraine \\ ${ }^{b}$ Princeton Plasma Physics Laboratory, P.O.Box 451, Princeton, NJ, 08543, USA
}

November 19, 1999

\begin{abstract}
The work summarizes results of the authors' studies on the energetic ion transport induced by sawtooth oscillations in tokamaks. The main attention is paid to description of physical mechanisms responsible for the transport. In addition to overview, the work contains new material. The new results concern the resonant interaction of the particles and the electromagnetic field of the sawtooth crash. In particular, it is discovered that the dominant harmonic of the crash $(m=n=1)$ can lead to stochastic motion of particles having large orbit width (potatoes). Regular motion of potatoes and quasi-stagnation particles in the presence of an $n=1$ mode is studied, and their characteristic displacements associated with quick switching on/off the mode are found.
\end{abstract}

*Invited paper presented in the 6th IAEA Technical Committee Meeting on Energetic Particles in Magnetic Confinement Systems, Naka, JAERI, 12-14 October, 1999 


\section{INTRODUCTION}

Sawtooth oscillations belong to the most typical forms of magnetohydrodynamic (MHD) activity in tokamaks. They are characterized by periodic collapses (crashes) of the pressure in the plasma core and play a crucial role in the formation of the temperature, density and current profiles. Because of this, since 1974, when they were discovered [1], the sawtooth oscillations have continuously stimulated extensive experimental and theoretical investigations.

Experiments, particularly on the large tokamaks (JET, TFTR), have convincingly shown that sawtooth oscillations may strongly affect the confinement of fast ions in the plasma core [2-10]. Observations have been made on ions over the wide range of energies: from a few tens of $\mathrm{keV}$ to $15 \mathrm{MeV}$. The fast ion sources were Neutral Beam Injection (NBI), Ion Cyclotron Resonance Frequency (ICRF) heating and fusion reactions.

Theoretical description of effects of sawtooth oscillations on fast ions is addressed in the present work. Its purpose is to make an overview basing on previous investigations of the authors and to present new results. The new results concern the resonant interaction of the dominant harmonic of the sawtooth crash $(m=n=1$, where $m$ and $n$ are the poloidal and toroidal mode numbers, respectively) and the particles satisfying the following two conditions. First, the particle energy, $\mathcal{E}$, must exceed the critical magnitude $\mathcal{E}_{\text {crit }}$ [11], i.e., the particles must be in the energy range in which their transport caused by the crash essentially depends on the pitch angle. Second, the toroidal drift velocity must be of the order of the longitudinal velocity, which implies that particles orbits are potato-like rather than banana-like. Note that such particles may constitute the main fraction of the energetic ion population in the region of sawteeth. The possibility of the resonant influence of the crash on potatoes was shown in previous works of the authors [12,13], where, however, higher harmonics $(n>1)$ of the crash, which are characterized by smaller amplitudes, were considered.

The work is organized as follows. The review material is included to Secs. II-IV, VI. In particular, Sec. II deals with earlier works, where it was assumed that fast ions are attached to (frozen in) the evolving magnetic flux surfaces. This section briefly describes the used crash models and the results of numerical simulation of the change of neutron emission during the crash in JET NBI experiments. Section III tells about first results on physical mechanisms involved in the crash-induced transport of fast ions. Section IV considers the resonant interaction of the particles and the higher harmonics of the crash. The new results are presented in Sec. V, where both regular and stochastic motion of energetic ions caused by the $m=n=1$ mode is studied. In addition, in Secs. IV, V, with using the developed theory, possible mechanisms for explanation of disappearance of strongly localized $\gamma$-ray and neutron emission region during crashes in ICRF heating experiments on JET are suggested. The energetic alpha particles

escaping to the wall because of sawtooth crashes in TFTR DT experiments are considered in Sec. VI. Finally, Sec. VII summarizes the conclusions.

\section{PARTICLES ATTACHED TO FLUX SURFACES. MODELLING NBI EXPERIMENTS ON JET}

When the energy of a fast ion is not very high, its behaviour during a sawtooth crash is similar to that of a thermal ion. To demonstrate this, let us present the particle guiding-centre 
velocity as

$$
\vec{v}=\vec{v}_{\|}+\vec{v}_{E}+\vec{v}_{D}
$$

where $\vec{v}_{\|}, \vec{v}_{E}$, and $\vec{v}_{D}$ describe the particle motion along the magnetic field, the $\vec{E} \times \vec{B}$ drift, and the toroidal drift, respectively. Assuming that the crash results from the $m=n=1$ instability, we introduce the helical co-ordinate system $\left(x^{1}, x^{2}, x^{3}\right)=(r, \alpha \equiv \vartheta-\varphi, \varphi)$, where $r, \vartheta$ and $\varphi$ are the radial, poloidal, and toroidal co-ordinates, respectively, chosen so that in the unperturbed state $B^{3} / B^{2}=q(r), B^{j}$ are contravariant components of the magnetic field strength. Then the perturbed electromagnetic field is described in terms of the helical magnetic flux $\Psi(r, \alpha, t)$ as follows:

$$
\begin{gathered}
\vec{B}=B^{3} \vec{e}_{3}+\vec{e}^{3} \times \nabla \Psi, \\
\vec{E}=\frac{1}{c} \frac{\partial \Psi}{\partial t} \vec{e}^{3}-\nabla \Phi
\end{gathered}
$$

where $\Phi(r, \alpha, t)$ is the electric potential associated with $\Psi$ due to the equation $\vec{E} \cdot \vec{B}=0$, which follows from the ideal MHD; $\vec{e}_{j}$ and $\vec{e}^{j}$ are the co- and contravariant basis vectors, respectively. One can see that the change of the helical magnetic flux along a particle orbit is given by

$$
\frac{d \Psi}{d t} \equiv \frac{\partial \Psi}{\partial t}+\vec{v} \cdot \nabla \Psi=c E_{3}+\left[\vec{v}_{E} \times \vec{B}\right]_{3}+O\left(v_{D}\right)
$$

Hence, taking into account that $c \vec{E}+\vec{v}_{E} \times \vec{B}=0$ when $\vec{E} \cdot \vec{B}=0$, we conclude that the particles follow the flux surfaces if the toroidal drift is negligible, the $\vec{E} \times \vec{B}$ drift being responsible for this. This implies that there is a possibility to simulate the transport of fast ions during the crash, assuming that they are attached to (or frozen in) the evolving flux surfaces.

For theoretical interpretation of the observed redistribution of fast ions, one needs to know the physics of the sawtooth crash. At present, there are different opinions on the nature of the sawtooth crash. The most widely known is the Kadomtsev model [14], which assumes that the crash results from magnetic field line reconnection caused by the $m=n=1$ kink instability. However, this model implies that the central safety factor, $q_{0}$, equals unity after the crash, which often contradicts to experimental data. For this reason, another reconnection model, which agrees with the experimentally observed small change of $q_{0}$ during the crash, was proposed [15]. According to this model, two distinct reconnection processes take place in two current layers, the first one joining flux surfaces together (as in the Kadomtsev model), and the second one splitting them. The "splitting" reconnection cuts off fragments of magnetic surfaces, pulled into the centre of the plasma, and creates a bubble-like formation with $q<1$. The possibility of such a new type of reconnection was confirmed in numerical calculations [16].

Based on the crash models of Refs. [14,15] and the theory of Ref. [17], where the fast ions were assumed to move together with evolving flux surfaces, a numerical simulation of the effect of crashes on injected deutrons with the energy of 80 and $140 \mathrm{keV}$ in JET NBI experiments was carried out [18]. In the mentioned experiments, the global and line-integrated neutron emission was measured and analysed tomographically to deduce the spatial distributions of the $2.5-\mathrm{MeV}$ neutron emission before and after the crash. The results show that during crashes 
peaked distributions are essentially changed, which implies expulsions of fast ions from the plasma core. In the simulations [18], the change of the local, line-integrated and global 2.5-MeV neutron emission associated with beam-plasma and beam-beam reactions was calculated. It was found that experimental results and numerical calculations are in reasonable agreement for both crash models [14,15], the results being sensitive to the $q(r)$ profile. To conclude which model describes the effects of crashes on the fast ions better, additional experimental data concerning $q(r)$ are required. But, in any case, the model [15] has the advantage of being consistent with persistence of the so-called "snakes" and other important experimental observations, whereas the Kadomtsev model is simpler and, thus, more convenient for analytical modelling of the evolving magnetic configuration.

Note that, according to Ref. [3], the post-crash distribution of injected ions in JET is hollow. Hollow post-crash distributions are easily reproduced in calculations employing reconnection models of the crash, which is demonstrated in Fig. 1, but can hardly be obtained in crash models assuming that plasma turbulence or magnetic field stochastization are the reason of the particle redistribution. On the other hand, in some experiments post-crash distributions of energetic ions were flat. In particular, circulating alpha particles with $0.15 \mathrm{MeV} \leq \mathcal{E} \leq 0.6 \mathrm{MeV}$ measured by $\alpha$-CHERS (CHarge Exchange Recombination Spectroscopy) diagnostics in TFTR DT experiments had flat post-crash profiles [5]. The approximation of flux-surface-attached particles was successfully used to model this experiment [5]. However, the same experimental data can be explained in the framework of another model as well [20]. According to Ref. [20], the particle mixing results from the combined action of the reconnection and the stochasticity of magnetic field lines, the stochasticity being caused by the presence of several modes with low mode numbers at the final stage of the sawtooth crash.

\section{THE CRITICAL ENERGY}

Although injected ions with $\mathcal{E} \sim 100 \mathrm{keV}$ in JET and circulating fusion-produced alpha particles with the energy from 150 to $600 \mathrm{keV}$ in TFTR can be described with the approach of Refs. $[17,18]$, it is clear that the basic assumption of Refs. $[17,18]$, that the particles are attached to the evolving flux surfaces, is rather artificial for superthermal ions. It is not justified for ions with $\Delta r_{b} \sim r_{\text {mix }}$, where $\Delta r_{b}$ is the particle orbit width in the absence of MHD perturbations, $r_{\text {mix }}$ is the sawtooth mixing radius. Furthermore, it may be not justified even for ions with $\Delta r_{b} \ll r_{m i x}$ because the toroidal precession leads to deflection of particles from the flux surfaces, which are not axisymmetric during the crash.

An essential contribution to understanding the physics of sawtooth-crash-induced transport of energetic ions was made in the work [11], which was first reported in the Fourth IAEA Technical Committee Meeting on Alpha Particles in Princeton (April 1995). In this work a new theory based on considering the interaction between particles and the evolving electromagnetic field of the crash was developed. One could say that "a new language" was suggested in Ref. [11]: instead of introducing the moving magnetic flux surfaces, the crash induced electric field following

from the ideal magnetohydrodynamics, $\vec{E}=-\vec{u} \times \vec{B} / c(\vec{u}$ is the plasma hydrodynamic velocity associated with the crash), was introduced. This led to the conclusion that the factor driving the particle redistribution is the $\vec{E} \times \vec{B}$ drift motion (see also Sec. II), whereas the toroidal 
precession tends to decorrelate the phase of interaction of particles and the electromagnetic helical perturbation associated with the crash. Another drawn conclusion was that the effect of sawtooth oscillations on particles with different energies and pitch angles may be different. This conclusion is based on the fact that the $\vec{E} \times \vec{B}$ drift motion dominates for slow ions, whereas the effects of the toroidal drift (precession, finite orbit width) and the longitudinal motion can be important for fast ions. Consequences of the joint effect of the toroidal precession and the perturbation on a particle depend on the comparative magnitudes of the characteristic times of the particle motion. Different behaviour of particles with $\tau_{p}>\tau_{c r}\left(\tau_{p}\right.$ is the characteristic time of the toroidal precession, $\tau_{c r}$ is the crash duration) and particles with $\tau_{p}<\tau_{c r}$ is shown in Fig. 2.

The most important result of Ref. [11] was the prediction of the critical energy, $\mathcal{E}_{\text {crit }}$. It was found that when $\mathcal{E}>\mathcal{E}_{\text {crit }}$, the bulk of trapped ions with small banana width are weakly sensitive to a crash, whereas circulating particles are strongly redistributed. In the contrary case, $\mathcal{E} \ll \mathcal{E}_{\text {crit }}$, both circulating and trapped particles are strongly redistributed by the crash, being "attached" to the evolving flux surfaces. Their redistribution can be described by the earlier theory $[17,18]$. The existence of $\mathcal{E}_{\text {crit }}$ immediately follows from Fig. 2 if one takes into account that $\tau_{p}$ depends on the particle energy. The critical energy is determined from the condition that the crash duration is equal to the period of the toroidal precession of a well-trapped ion. It can be written as follows:

$$
\mathcal{E}_{\text {crit }}=2 \pi M k_{s} r_{s} R_{0} \omega_{B} / \tau_{c r}
$$

where $k$ is the ellipticity of the cross section, the subscript " $s$ " labels the magnitudes at the $q=1$ flux surface, $M$ is the ion mass, $\omega_{B}$ is the cyclotron frequency, $R_{0}$ is the major radius of the torus. When $\tau_{c r} \sim 10^{-4} \mathrm{~s}, \mathcal{E}_{\text {crit }}=300 \div 700 \mathrm{keV}$ for $\alpha$-particles in TFTR and JET.

The existence of $\mathcal{E}_{\text {crit }}$ was confirmed both numerically and experimentally.

In particular, a numerical simulation of $\alpha$-particle behaviour in a JET-like tokamak showed that redistribution of trapped and circulating alphas with $\mathcal{E}>\mathcal{E}_{\text {crit }}$ is very different; in addition, it showed that the effect of crashes on trapped alphas essentially depends on the particle energy when the latter varies from $280 \mathrm{keV}$ to $2.8 \mathrm{MeV}$, see Figs. 3 and 4 [21,22].

Decrease of the sawtooth-induced redistribution of $\alpha$-particles with the particle energy was observed in DT experiments on TFTR, where the behaviour of well-trapped alphas with the energy from $0.64 \mathrm{MeV}$ to $1.21 \mathrm{MeV}$ during sawteeth was studied, using PCX (Pellet Charge eXchange) diagnostics [6]. Later, analyses of those data presented in Refs. [7-9] showed that the experimental results can be well interpreted in terms of the existence of the critical energy according to Eq. (5).

Furthermore, analysing the experimental data from JET and TFTR [3-5,10], one can conclude that, when $\mathcal{E}>\mathcal{E}_{\text {crit }}$, circulating particles are much more sensitive to the crash than trapped ones, which agrees with the theory of Refs. [11,21,22]. 


\section{REDISTRIBUTION OF IONS WITH $\mathcal{E}>\mathcal{E}_{\text {crit }}$ CAUSED BY UNPERTURBED RESONANCES}

\section{A. Resonant particles}

There are certain groups of trapped particles which can be essentially affected by the crash even when $\mathcal{E}>\mathcal{E}_{\text {crit }}$. These are resonant particles, whose displacements by the perturbation on different bounce periods do not cancel each other. This can be shown as follows. Assuming that $\tau_{c r} \gg \tau_{b}$, where $\tau_{b}$ is the particle bounce period, let us take a perturbed quantity in the form $X=\hat{X}(r, t) \exp (i n \vartheta-i m \varphi)$, where $\hat{X}$ slowly varies in time. Then, using the fact that when $\hat{X}$ is small, $\langle\dot{r}\rangle \propto X$, where $\langle(\ldots)\rangle$ denotes bounce averaging, we estimate the particle displacement during the crash as

$$
\int_{0}^{\tau_{c r}}\langle\dot{r}\rangle d t \propto \sum_{p=0}^{N} \hat{X} \exp \left(-i p n \omega_{\varphi} \tau_{b}\right)+\langle\delta r\rangle
$$

where $\omega_{\varphi}=\langle\dot{\varphi}\rangle$ is the precession frequency, $N$ is the integer part of the ratio $\tau_{c r} / \tau_{b}, p$ is an integer, $\langle\delta r\rangle$ is the particle displacement for $\Delta t<\tau_{b}$. It follows from Eq. (6) that the particle displacement is maximum when either $\omega_{\varphi} \tau_{c r} \ll 2 \pi$, which corresponds to the case of $\mathcal{E}<\mathcal{E}_{\text {crit }}$, or the following resonance condition is satisfied $[12,13]$ :

$$
s \omega_{b}=n \omega_{\varphi}
$$

where $\omega_{b}$ is the bounce frequency, $s$ is an integer. For the helical perturbation $(m=n)$, Eq. (7) determines the resonant values of $\mathcal{E}$ and $\mu$ at a given $r, \alpha \equiv \vartheta-\varphi$ and a resonant drift surface $r(\alpha)$ for a particle with given $\mathcal{E}, \mu$. The particles that are exactly in the resonance are characterized by vanishing change of energy $(\langle\dot{\mathcal{E}}\rangle \approx-e\langle\dot{\Phi}\rangle=0)$.

In order to see which particles are resonant, it is convenient to give topological classification of particles with arbitrary orbit width in the low-shear region in terms of two dimensionless constants of motion: $P=-2 c P_{\varphi} q /\left(k e B_{0} R_{0}^{2} \nu^{2}\right)$ (where $P_{\varphi}$ is the toroidal canonical angular momentum, $B_{0}$ is the magnetic field at the magnetic axis) and $L=(1-\lambda) \nu^{-1}$, which characterizes the particle pitch angle [here $\lambda=\mu B_{0} / \mathcal{E}, \mu$ is the particle magnetic moment, $\nu=\left[2 q \rho /\left(k R_{0}\right)\right]^{2 / 3}$, $\left.\rho=v / \omega_{B}, v=(2 \mathcal{E} / M)^{1 / 2}\right][25,13]$. This classification is presented in Fig. 5. The level contours of the ratio of the frequencies of the toroidal and poloidal motion are shown in Fig. 6 for $\nu=0.11$, the results being weakly sensitive to the magnitude of $\nu$.

The pictures shown in Figs. 5, 6 are applicable to the sawtooth mixing region where $|q-1| \ll$ 1. Therefore, we conclude that in this region the ratio $\omega_{\varphi} / \omega_{b}$ is slightly less than unity for potatoes, whereas $\omega_{\varphi} / \omega_{b} \ll 1$ for narrow bananas.

Note that studying behaviour of potatoes and other particles with non-standard orbits is of importance because they may constitute the main fraction of the energetic ion population in the region of sawteeth. This becomes clear if one takes into account that these particles are located inside the radius $r \sim \nu(\mathcal{E}) R$, which may be of the same order as $r_{m i x}$.

One can single out two situations when Eq. (7) can be satisfied. First, the particles that do not undergo the toroidal precession $\left(\omega_{\varphi}=0\right)$ are resonant with modes of any $n$. As the $m=n=1$ mode and its low- $n$ satellites dominate the sawtooth crash, this is the only resonance possible for trapped particles with narrow orbits. Second, resonances with $n \sim 1(n \neq 1)$, which are possible for potato particles. These situations are considered in Subsec. IV B and IV C. 


\section{B. Superbananas arising due to weak precession}

It is known that the toroidal precession of marginally trapped particles with the narrow orbit width can be rather weak. In particular, when the magnetic shear is small, the precession vanishes for the particles with the trapping parameter $\kappa \approx 0.9\left(\kappa=\left(\mathcal{E}-\mu B_{0}(1-\epsilon) /\left(2 \epsilon \mu B_{0}\right)\right.\right.$, $\epsilon=r / R)$. This implies that the factor competing with the $\vec{E} \times \vec{B}$ drift is weak for such particles, and thus, the crash will affect them even when $\mathcal{E}>\mathcal{E}_{\text {crit }}$. On the other hand, the crash-induced radial motion changes $\kappa$, which increases the precession velocity. The direction of the toroidal precession is different inside and outside the radius where the precession is vanishing. Therefore, crash may result in superbanana motion of marginally trapped particles around the surface where $\kappa \approx 0.9$ Ref. [23].

In order to demonstrate the appearance of superbananas associated with the electromagnetic field of a crash, we adopt Eqs. (2), (3) for the electromagnetic field and assume that $\tau_{b} \ll \tau_{c r}$ and $|q-1| \ll 1$ in the sawteeth mixing region. This enables us to employ the averaging procedure described in Ref. [21] for deriving the equations of the bounce averaged drift motion. These equations can be written in the Hamiltonian form as follows:

$$
\dot{P}_{\varphi}=-\frac{\partial H}{\partial \alpha}, \quad \dot{\alpha}=\frac{\partial H}{\partial P_{\varphi}}
$$

where $H=H\left(P_{\varphi}, \alpha, J_{\|}\right)$is the Hamiltonian, $P_{\varphi}$ and $\alpha$ play the part of canonical variables, $J_{\|}$ is the longitudinal adiabatic invariant.

In the absence of MHD perturbations $H=H_{0}\left(P_{\varphi}, J_{\|}\right)$, so that Eq. (8) describes the toroidal precession at $r=$ const with the rate $\dot{\alpha}=\omega_{\varphi 0} G(\kappa)$, where $\omega_{\varphi 0}$ is the precession frequency of particles with $\kappa \ll 1, G(\kappa)=2 E(\kappa) / K(\kappa)-1, K(\kappa)$ and $E(\kappa)$ are the complete elliptic integrals of the first and second kind, respectively. The equation $G(\kappa)=0$ has a root, $\kappa_{r} \approx 0.91$.

In the presence of a perturbation the Hamiltonian can be written in the form $H=H_{0}+$ $e \Phi\left(P_{\varphi}\right) \cos \left[\alpha-\alpha_{0}\left(P_{\varphi}\right)\right]$, where $\Phi \sim E r_{s} \sim r_{s} v_{E} B / c \sim r_{s}^{2} B /\left(c \tau_{c r}\right), v_{E}$ is the $\vec{E} \times \vec{B}$ drift velocity (we used the fact that $v_{E} \tau_{c r} \sim r_{s}$ ). Let us expand this Hamiltonian in a power series in $P_{\varphi}-P_{\varphi r}$, where $P_{\varphi r}$ is the resonant magnitude of $P_{\varphi}$, at which $\kappa=\kappa_{r}$. Keeping terms of $H_{0}$ up to the second order and only the zero-order term of the perturbation potential, we obtain the wellknown Hamiltonian of the non-linear pendulum:

$$
H=\frac{1}{2} \omega_{\varphi r}^{\prime}\left(P_{\varphi}-P_{\varphi r}\right)^{2}+e \Phi_{r} \cos \left[\alpha-\alpha_{0}\left(P_{\varphi r}\right)\right]
$$

where $\Phi_{r} \equiv \Phi\left(P_{\varphi r}\right), \omega_{\varphi r}^{\prime} \equiv \partial \omega_{\varphi} /\left.\partial P_{\varphi}\right|_{P_{\varphi r}}$, and the constant term $H_{0}\left(P_{\varphi r}\right)$ was omitted. The lines of level of Eq. (9) near the resonance form islands, which correspond to superbanana orbits. The particles within the island are trapped with respect to the perturbation phase. Deviation of a particle from $P_{\varphi r}$ caused by the perturbation results in "detuning" of the particle from the wave because it leads to $\omega_{\varphi} \neq 0$. The change of the perturbation phase prevents further radial displacement and then reverses the direction of the displacement.

The physical nature of the resonance island at $\kappa=\kappa_{r}$ resembles that of the superbanana orbits in stellarators [24]. Particles trapped in a helical magnetic well of a stellarator undergo precession along the well. Like in the case considered above, the precession vanishes for particles with $\kappa=\kappa_{r}$ [note that the definition of the trapping parameter, $\kappa$, for a helically trapped particle 
in a stellarator is, of course, other than for toroidally trapped one in a tokamak]. The toroidicity plays the role of a perturbation. The resulting pattern of the bounce-averaged particle motion is similar to that described by the Hamiltonian (9). Wide trajectories arising near the resonance $\kappa=\kappa_{r}$ are located at the inner circumference of the torus and called superbananas. Their width is proportional to the square root of the perturbation and exceeds width of trajectories for $\kappa \neq \kappa_{r}$. The physical mechanism creating the superbananas in stellarators coincides with the one described above. Namely, the perturbation results in radial deviation from the resonance, which, in turn, leads to "detuning" from the resonance and reversing the radial motion.

Thus, although the shape and location of trajectories of superbananas in stellarators and the considered resonant particles in tokamaks differ, their origin and essential features are, in fact, the same. For this reason, we call the orbits of the resonant particles during the crash as "superbanana orbits".

The superbanana width, $\Delta r_{s b}$, and the period of the superbanana motion, $\tau_{s b}$, can be estimated as

$$
\begin{gathered}
\Delta r_{s b} \simeq 4\left|\frac{2 c \Phi_{r}}{B_{0} \omega_{\varphi 0}}\right|^{1 / 2} \sim \frac{8 r_{s}}{\left(2 \tau_{c r} \omega_{\varphi 0}\right)^{1 / 2}}, \\
\tau_{s b}=\frac{\Delta r_{s b}}{r_{s}} \tau_{c r}
\end{gathered}
$$

where $\left(\tau_{c r} \omega_{\varphi 0}\right)^{1 / 2} \gg 1$ when $\mathcal{E} \gg \mathcal{E}_{\text {crit }}$. The equation (10) shows that the width of the superbananas produced by the crash depends on the particle energy (unlike the superbanana width in stellarators). Both $\Delta r_{s b}$ and $\tau_{s b}$ decrease with $\mathcal{E}$. When $\mathcal{E} \gg \mathcal{E}_{\text {crit }}$ then $\tau_{s b} \ll \tau_{c r}$, and, therefore, the crash forms plateaus with the width of the order of $\Delta r_{s b} \ll r_{s}$ near the resonance radii where $P_{\varphi}=P_{\varphi r}$. When $\mathcal{E} \leq \mathcal{E}_{\text {crit }}$ then the superbanana width is as large as the sawtooth mixing region, but $\tau_{s b} \geq \tau_{c r}$. In the latter case, which can be considered as a degenerate one (all particles are resonant), the post-crash distribution of fast ions is hollow rather than flat (if the pre-crash state is peaked), see Sec. II.

\section{Superbananas with strong toroidal precession}

For the particles satisfying Eq. (7), the toroidal precession does not decorrelate the phase of the wave-particle interaction. Therefore, they can strongly interact with the electromagnetic perturbation in spite of the fact that they are characterized by strong toroidal precession [23,13]. But it is clear that in order to satisfy Eq. (7) the presence of corresponding harmonics in the electromagnetic field of the crash is required.

A sawtooth crash is presumably associated with development of the $m=n=1$ helical perturbation. Nevertheless, higher harmonics with $m=n$ are to be present, too. The reason is that the perturbation having initially the sinusoidal form becomes non-harmonic in the nonlinear stage of the instability. In particular, for a Kadomtsev-type crash, the amplitudes of the harmonics with $m=n=2,3$ are $0.2 \div 0.6$ the amplitude of the $m=n=1$ mode [13]. The higher modes $(m=n \geq 4)$ are less important.

The presence of harmonics with $n>1$ provides the resonant interaction between the crash and the particles with non-standard orbits (potatoes, quasi-stagnation orbits etc). 
The motion of large orbit potatoes near the resonances under influence of a perturbation was studied in Ref. [13] with using two approaches. First, the guiding center code OFSEF (Orbit Following in the Sawtooth Electromagnetic Field) was used to construct a Poincaré map of the particle motion near a resonance. Second, the width of superbananas and the period of the superbanana motion were calculated via a derived adiabatic invariant of the resonant motion (see also [26]).

Examples of particle orbits are given in Fig. 7 showing intersections of particle orbits with the equatorial plane of the torus. The orbits presented in this figure result from an electrostatic $m=n=3$ perturbation of the form $\Phi=\Phi_{\max } f_{3}(r) \sin (3 \alpha)$, where the function $f_{3}(r)=5.38\left(r / r_{\text {mix }}\right)^{3}\left[1-\left(r / r_{\text {mix }}\right)^{2}\right], \max f_{3}(r)=1$, models the radial profile of the perturbation. One can see the resonance island (superbananas) produced by an $m=n=3$ electrostatic perturbations near the resonance $\omega_{\varphi} / \omega_{b}=2 / 3$. The superbanana motion, just as in the case of $s=0$ superbananas, can be expected to produce plateaus in the phase space on the fast ion distribution function. The width of such plateaus in the physical space, as one can see from Fig. 7 is not large (less than the width of the unperturbed orbits). However, the resonance islands of the resonances $1 / 3,1 / 2$, and $2 / 3$ overlap for MeV-range ions at perturbation amplitudes typical for the crash, which can lead to stochastic motion of a noticeable fraction of the potato ions [13].

\section{Hot-spot experiment on JET}

Different behaviour of the resonant and non-resonant particles may account for results of the experiment with ICRF minority heating on JET where strongly localized gamma ray and neutron emitting region (the "hot spot"), observed before the crash, disappeared after the crash. This experiment was described in Ref. [10]. According to Ref. [10], the population of energetic particles consisted of two groups of accelerated ${ }^{3} \mathrm{He}$ ions. The largest group consisted of trapped particles having the turning points in the cyclotron resonance layer and the energy about 2 $\mathrm{MeV}$. The other group was very small and included particles passing in the toroidal direction and strongly localized poloidally in the vicinity of the equatorial plane of the torus near the magnetic axis, where the hot spot was located. The estimated energy of the hot-spot ions was several megaelectronvolts. Following Ref. [10], we assume that the typical fast ions are trapped particles with the banana tips in the vicinity of the ICRF resonance layer. Concerning the hot-spot ions, we assume that they have orbits that are well localized in the poloidal and radial directions near the equatorial plane of the torus, where the hot spot is located. Such orbits exist for ions with very small pitch angles and belong to semi-trapped particles that are trapped poloidally but untrapped toroidally, i.e., they are of quasi-stagnation type.

Allowing for these facts, we take $\mathcal{E}=2 \mathrm{MeV}, \lambda=1$, and the banana tip radius $r_{t}=28 \mathrm{~cm}$ for a typical fast ion. Then we obtain that $L=0, P=1.1$, which implies that the typical ion is outside of the region of resonances with $n=1 \div 3$ important for the crash resulting from the $m=n=1$ instability. For this reason, one can expect that the crash will weakly affect the bulk of fast ions. A numerical simulation with the guiding-center code OFSEF, which employs the analytical expression of the electromagnetic field perturbation modeling the Kadomtsev-type crash [11], confirms this, see Fig. 8. 
As examples of the hot-spot ions, we take the ions with $\mathcal{E}=2 \div 5 \mathrm{MeV}$, the pitch angle of $100^{\circ}$, and $R-R_{0}=20 \mathrm{~cm}$. We find that they may be resonant, being located between the $s / n=1 / 2$ and $s / n=1$ resonances on the $(L, P)$ plane. Generated Poincaré maps confirm that these particles are indeed resonant and have large width of the superbanana orbits (see Fig. 9). In addition, direct numerical simulation of the particle behavior during a crash with the code OFSEF shows that the the hot-spot ions are expelled well outside the region where they were located before the crash, which implies disappearance of the hot spot. These results are shown in Fig. 10.

Thus, direct numerical simulations of particle behaviour in a Kadomtsev-type crash with the code OFSEF showed that the redistribution of hot-spot particles, in contrast to banana particles, is indeed strong enough to explain the disappearance of the spot [27]. However, such a simulation still leaves a question on the mechanisms of such redistribution. A possible explanation is the particle redistribution due to the superbanana motion analysed in this section. It was shown that a $m=n=2$ harmonic with the amplitude typical for a sawtooth crash in JET leads to strong superbanana motion of hot-spot ions, and the width of the superbananas is sufficient to account for the observed redistribution [13]. Moreover, joint action of the harmonics $m=n=2$ and $m=n=3$ results in stochastic motion of the particles, but the time of the crash may be not sufficient for the stochastic diffusion to manifest itself. One cannot rule out that other mechanisms contribute to the redistribution of the hot-spot ions, which will be considered in the next section.

\section{FORCED RESONANCES AND MOTION OF IONS WITH $\mathcal{E}>\mathcal{E}_{\text {crit }}$}

In spite of the fact that the exact resonance condition for the interaction of particles and the dominant $n=1$ harmonic of the crash cannot be satisfied, one can expect that their resonant interaction is possible due to forced resonances. The matter is that, as will be shown below, the presence of the perturbation results in forced resonance $\omega_{\varphi}=\omega_{b}$ and resonances $s \omega_{b}^{\prime}=n^{\prime} \omega_{\varphi}^{\prime}$ (primes refer to the co-ordinate system associated with the perturbed motion of a particle), which arise due to the appearance of harmonics with higher toroidal mode numbers in the perturbed system. These resonances are mainly important for the particles for which the ratio $\omega_{\varphi} / \omega_{b}$ is only slightly less then unity in the unperturbed state, i.e., for particles with potato and stagnation orbits. The analysis below deals with such particles.

\section{A. Motion in SARD}

A distinctive feature of the particles with non-standard orbits is that they are characterized by the poloidal action integral $J_{\theta} \equiv \oint d \vartheta P_{\vartheta} / 2 \pi\left(P_{\vartheta}\right.$ is the poloidal canonical momentum [28], $\vartheta$ is the poloidal angle) which is small compared to that of the narrow-orbit circulating and trapped (banana-like) particles, tending to zero when the orbit approaches the stagnation orbit. Therefore, below we will refer to this particles as Small Action Particles (SAP).

To study the particle guiding-center motion, we use action-angle variables of the unperturbed system, proceeding from the following Hamiltonian:

$$
H\left(J_{\theta}, J_{\phi}, \theta, \phi\right)=H^{(0)}\left(J_{\theta}, J_{\phi}\right)+V\left(J_{\theta}, J_{\phi}, \theta, \phi\right)
$$


where $H^{(0)}$ is the Hamiltonian of the motion in the absence of the perturbation; $\theta=\theta(\vartheta)$ and $\phi=$ $\varphi+\chi(\vartheta)$ are the canonical poloidal and toroidal angles $[29,13] ; \chi(\vartheta)$ is a periodic function; $J_{\theta}$ and $J_{\phi} \equiv \oint d \varphi P_{\varphi} / 2 \pi$ are the corresponding action variables; $V=\sum_{s} V_{s}\left(J_{\theta}, J_{\phi}\right) \cos (s \theta-\phi)$ is an $n=$ 1 perturbation. The existence of many harmonics in $\theta$ is a consequence of the toroidicity of the system resulting in non-uniformity of the particle motion in the poloidal direction. This should not be confused with MHD mode coupling due to toroidicity, which often leads to appearance of the sidebands with the poloidal mode number $m+1$ and $m-1$ and the amplitude $\sim A^{-1}$. The non-uniformity of the poloidal motion of trapped particles is $\sim 1$; therefore, the Fourier spectrums of a perturbation in $\vartheta$ and $\theta$ are completely different. In particular, a quasi-stagnation particle almost does not "feel" the poloidal (in $\vartheta$ ) structure of the mode because the poloidal angle almost does not vary during its motion. For such a particle, the harmonics with $s=0$ and $s=1$ dominate.

At first, allowing for the fact that quasi-stagnation and potato particles are characterized by small $J_{\theta}$, we restrict ourselves to the system linearized near $J_{\theta}=0$ and a certain $J_{\phi}=J_{\phi 0}$. Then we can write $H^{(0)}=\omega_{\theta} J_{\theta}+\omega_{\phi}\left(J_{\phi}-J_{\phi 0}\right)$, where $\omega_{\theta}=\omega_{b}$ and $\omega_{\phi}=\omega_{\varphi}$ are the frequencies of the unperturbed motion. We consider only the $s=0$ and $s=1$ harmonics of the perturbation and take $V_{0}=$ const and $V_{1}=C J_{\theta}^{1 / 2}$. The latter can be justified as follows. First, the perturbed part of Hamiltonian is expected to be proportional to the generalized co-ordinates. Second, an action $J=\oint p d q$ is proportional to the square of the contour size when the contour shrinks to a point. The simplified system is integrable. Performing the canonical transformation $\hat{\theta}=\theta$, $\hat{\phi}=\theta-\phi, \hat{J}_{\theta}=J_{\theta}+J_{\phi}+V_{0} \omega_{\phi}^{-1} \cos \phi, \hat{J}_{\phi}=-J_{\phi}-V_{0} \omega_{\phi}^{-1} \cos \phi$, and omitting the constant term $\omega_{\phi} J_{\phi 0}$ we obtain the following new Hamiltonian:

$$
\begin{aligned}
\hat{H}\left(\hat{J}_{\theta}, \hat{J}_{\phi}, \hat{\theta}, \hat{\phi}\right) & =\hat{\omega}_{\theta} \hat{J}_{\theta}+\hat{\omega}_{\phi} \hat{J}_{\phi} \\
& +C\left(\hat{J}_{\theta}+\hat{J}_{\phi}\right)^{1 / 2} \cos \hat{\phi}
\end{aligned}
$$

where $\hat{\omega}_{\theta}=\omega_{\theta}, \hat{\omega}_{\phi}=\omega_{\theta}-\omega_{\phi}$. As $\partial \hat{H} / \partial \hat{\theta}=0, \hat{J}_{\theta}$ is a constant of motion. The level contours of the Hamiltonian (13) for a given $\hat{J}_{\theta}$ are shifted circles in the co-ordinates $\left(\hat{J}_{\phi}-\hat{J}_{\phi 0}\right)^{1 / 2} \cos \hat{\phi}$, $\left(\hat{J}_{\phi}-\hat{J}_{\phi 0}\right)^{1 / 2} \sin \hat{\phi}$, where $\hat{J}_{\phi 0}=-\hat{J}_{\theta}$ is the value of $\hat{J}_{\phi}$ that corresponds to $J_{\theta}=0$, i.e., the stagnation orbit. Note that $\hat{J}_{\phi}-\hat{J}_{\phi 0}=J_{\theta}$. The center of the shifted circles is located at the fixed point of the Hamiltonian, $J_{\theta}^{1 / 2}=C /\left(2 \hat{\omega}_{\phi}\right)$. At this point

$$
\dot{\hat{\phi}}=\dot{\theta}-\dot{\phi}=0
$$

i.e., driven resonance between the SAP and the $s=n=1$ harmonic takes place, which we will refer to as Small-Action Resonance (SAR). It is clear that there is no threshold for perturbation amplitude for SAR to occur. The circle with the radius $C /\left(2 \hat{\omega}_{\phi}\right)$ restricts the region where the particles are trapped with respect to the perturbation phase, which we will refer to as SmallAction Resonance Domain (SARD). The SARD, in fact, is produced by a simple shift of the vicinity of the O-point of the unperturbed system. Note that SARD typically arises in any integrable Hamiltonian system in the region of the phase space where, at least, one of the action variables is small [30].

The motion around SARD may result in radial redistribution of particles only in the case of fast switching on/off the perturbation. Otherwise, when the process begins and terminates 
slowly, the particles return almost to their initial radial positions after the perturbation disappears because the motion is adiabatic. Thus, the temporal evolution of the perturbation is crucial to determine the redistribution of SAPs due to their motion around SARD.

In order to demonstrate this, let us first consider the case when the characteristic time of the change of the perturbation amplitude is negligible in comparison with the period of the particle motion in SARD $\left(\tau_{S A R D}=2 \pi /\left(\omega_{b}-\omega_{\varphi}\right)\right)$, i.e. the perturbation is sharply switched on/off. To estimate the maximum displacement of a SAP caused by a $s=1$ perturbation, which we denote as $\Delta r_{S A R D}$, we use the fact that a particle located initially at $J_{\theta}=0$ (at the stagnation orbit) moves around SARD, reaching the maximum magnitude of $J_{\theta}$ equal to $J_{\theta}=C^{2} / \hat{\omega}_{\phi}^{2}$. Then, taking into account that $J_{\theta}=e B \delta r^{2} /(2 c)$ for quasi-stagnation particles, where $\delta r$ is the radius of the orbit in the poloidal cross section, we find:

$$
\Delta r_{S A R D}=\left(\frac{2 c}{e B} J_{\theta}\right)^{1 / 2}=\left(\frac{2}{M \omega_{B}}\right)^{1 / 2} \frac{C}{\omega_{b}-\omega_{\varphi}}
$$

(note that the estimate is correct only to the order of magnitude when the displacement is so large that the orbit turns into a potato). In the general case, we find the following estimate for the radial displacement:

$$
\frac{\Delta r}{\Delta r_{S A R D}} \sim \min \left\{1, \frac{\tau}{\tau_{S A R D}}\right\}
$$

where $\tau$ is the duration of the perturbation.

Now we analyse the case when $C$ varies gradually. We notice that the motion in $\hat{\phi}$ is separated from the motion in $\hat{\theta}$ and treat the co-ordinates $\hat{J}_{\phi}, \hat{\phi}$ as the only degree of freedom and $\hat{J}_{\theta}$ as a constant. Then the variables $\xi=\left[2\left(\hat{J}_{\phi}+\hat{J}_{\theta}\right)\right]^{1 / 2} \cos (\hat{\phi}), \eta=\left[2\left(\hat{J}_{\phi}+\hat{J}_{\theta}\right)\right]^{1 / 2} \sin (\hat{\phi})$ are other canonical co-ordinates, in which the Hamiltonian (13) takes the form

$$
H=\frac{\hat{\omega}_{\phi}}{2}\left(\xi^{2}+\eta^{2}\right)+\frac{C(t)}{2^{1 / 2}} \xi
$$

where we have omitted constant terms. When $C(t)$ is parabolic, $C(t)=4 C_{\max }(t / \tau)(1-t / \tau)$, the canonical transformation

$$
\begin{aligned}
& \xi_{*}=x+\frac{C}{2^{1 / 2} \hat{\omega}_{\phi}}+\frac{2^{5 / 2} C_{\text {max }}}{\hat{\omega}_{\phi}^{3} \tau^{2}} \\
& \eta_{*}=y-\frac{2^{3 / 2} C_{\max }}{\hat{\omega}_{\phi}^{2} \tau}\left(1-\frac{2 t}{\tau}\right)
\end{aligned}
$$

enables us to arrive at the Hamiltonian

$$
H_{*}=\frac{\hat{\omega}_{\phi}}{2}\left(\xi_{*}^{2}+\eta_{*}^{2}\right)
$$

which does not depend on time and, thus, is a constant of motion. Equation (20) describes rotation of particles around a centre shifted according to Eqs. (18), (19). The displacement of a stagnation particle $(\xi=0, \eta=0)$ at $t=\tau$ depends on the phase of the rotation and satisfies 
the inequality $0 \leq \Delta J_{\theta} \leq 16 C_{\text {max }}^{2} \tau^{-2} \hat{\omega}_{\phi}^{-4}\left(1+4 \tau^{-2} \hat{\omega}_{\phi}^{-2}\right)$. Assuming that $\tau \gg \tau_{S A R D}$ and using Eq. (15) and the relationship between $J_{\theta}$ and $\delta r$, we obtain:

$$
\frac{\Delta r}{\Delta r_{S A R D}\left(C_{\max }\right)} \leq \frac{2}{\pi} \frac{\tau_{S A R D}}{\tau}
$$

Thus, the displacement tends to 0 when $\tau \rightarrow \infty$.

Let us estimate $\triangle r_{S A R D}$ for the electric component of the crash perturbation. Estimating $V_{1}$ as $e E \delta r$ and using $V_{1}=C \delta r(e B / 2 c)^{1 / 2}$, we find $C=e E(2 c / e B)^{1 / 2}$. Then Eq. (15) yields:

$$
\Delta r_{S A R D} \sim \frac{2 r_{m i x}}{\tau_{c r}\left(\omega_{b}-\omega_{\varphi}\right)}
$$

When deriving this equation, we assumed that $v_{E} \sim r_{m i x} / \tau_{c r}$.

The presence of the $s=0$ harmonic changes the particle energy and results in an additional radial displacement, $\Delta r_{0}$. However, $\Delta r_{0}$ is small in comparison with $\Delta r_{S A R D}$. Furthermore, the $s=0$ harmonic does not lead to orbit transformation ( $J_{\theta}$ is not affected by this harmonic), and, as one can show, its effect on quasi-stagnation particles is even absent in the case of magnetic perturbations.

\section{B. Stochastization of potatoes}

To study non-linear effects, we return to the Hamiltonian (12) and look for action-angle co-ordinates of the perturbed system. In other words, we proceed to the perturbed co-ordinate system centered in the SAR point, i.e., to the co-ordinate system in which SAR does not exist. Considering the perturbation amplitude to be of the order of a small parameter $\varepsilon \ll 1$, we write $V=\varepsilon \sum_{s} \tilde{V}_{s}\left(J_{\theta}, J_{\phi}\right) \cos (s \theta-\phi)$. Then we use the perturbation technique [31] to find the canonical Lie transformation that annihilates the angle-dependent terms. To the first order in $\varepsilon$, the generator of the required transformation is produced by the following scalar generating function (the explicit expressions for the transformed co-ordinates in terms of the generating functions can be found in Refs. [31,32]):

$$
S\left(J_{\theta}, J_{\phi}, \theta, \phi\right)=-\sum_{s} \frac{\tilde{V}_{s}}{s \omega_{\theta}-\omega_{\phi}} \sin (s \theta-\phi)
$$

where $\omega_{\theta}=\partial H^{(0)} / \partial J_{\theta}, \omega_{\phi}=\partial H^{(0)} / \partial J_{\phi}$. The denominators in Eq. (23) reflect the fact that the required transformation would not exist near resonances as the drift tori would be destroyed there. However, since $\omega_{\theta} \geq \omega_{\phi}$, such resonances do not occur. The Hamiltonian in the new co-ordinates takes the form

$$
\begin{aligned}
& H=H^{(0)}\left(J_{\theta}^{\prime}, J_{\phi}^{\prime}\right)+\varepsilon^{2}\left[f_{1}\left(J_{\theta}^{\prime}, J_{\phi}^{\prime}, \theta^{\prime}\right)\right. \\
& \left.+f_{2}\left(J_{\theta}^{\prime}, J_{\phi}^{\prime}, \theta^{\prime}\right) \cos \left(2 \phi^{\prime}\right)+f_{3}\left(J_{\theta}^{\prime}, J_{\phi}^{\prime}, \theta^{\prime}\right) \sin \left(2 \phi^{\prime}\right)\right]
\end{aligned}
$$

We see that the modes with $n=2$ and $n=0$ appear in the second order. The structure of the functions $f_{2}$ and $f_{3}$ (which are not presented here) shows that the non-linearity of the system and/or the presence of higher harmonics in $\theta$ (but not in $\vartheta$ ) are important for the $n=2$ harmonic 
to arise (otherwise, as we have shown above, the system is integrable). The procedure can be extended to next orders, where harmonics with $n>2$ are to appear.

Thus, the break of the toroidal symmetry produced by the $n=1$ mode of finite amplitude makes this mode to be non-monochromatic in the canonical toroidal angle of the perturbed system $\left(\phi^{\prime}\right)$. The appearance of higher harmonics in the perturbed system is of great importance because it makes possible multiple resonance islands due to interaction of SAPs with the $n=1$ perturbation through the resonances $s^{\prime} \omega_{\theta}^{\prime}=n^{\prime} \omega_{\phi}^{\prime}$, where $s^{\prime}$ and $n^{\prime}$ are the harmonic numbers in the perturbed system, which is likely to result in stochastic motion when the perturbation amplitude exceeds a certain magnitude.

In order to study this process, we generated numerically Poincaré maps of the particle motion in a tokamak in the presence of a steady-state $m=n=1$ perturbation, using the guidingcenter code OFSEF. We assumed that the perturbation was either magnetic, $\vec{B}=\nabla \varphi \times \nabla \Psi$, or electrostatic, $\vec{E}=-\nabla \Phi$, with the potentials $\Psi$ and $\Phi$ given by $X=X_{\max } 4\left(r / r_{\max }\right)(1-$ $\left.r / r_{\text {max }}\right) \exp (i \vartheta-i \varphi)$, where $X$ is either $\Psi$ or $\Phi, r_{\text {max }}$ is the radius inside which the perturbation is localized, $X_{\max }$ is the maximum of $|X|$. Calculations were carried out for ${ }^{3} \mathrm{He}$ ions of various energies and magnetic moments in JET in the presence of perturbations of various amplitudes. We found that, starting from a certain amplitude, motion of some group of particles becomes stochastic. Figure 11, where the effect of a magnetic perturbation on 1-MeV ions with quasistagnation, potato, and banana orbits is shown, demonstrates this. We observe that there two zones of regular motion separated by a region of stochasticity. The inner zone corresponds to quasi-stagnation particles; the outer one, to bananas. The region of stochasticity consists of potato particles. It was found from more detailed analysis that the stochasticity arises from overlap of resonance islands with $n^{\prime}=2$ and $n^{\prime}=3$. Increase of the amplitude leads to expansion of the stochasticity to the region of quasi-stagnation orbits, whereas banana particles remain almost unaffected by stochasticity. The expansion of the stochasticity is rather rapid: the change of the amplitude required for the stochasticity to spread over the whole region of non-standard orbits is only by a factor about 1.5 from the level corresponding to Fig. 11). Therefore, we can say that there is an amplitude threshold of the appearance of the stochasticity. Calculations carried out for various $L$ give similar results, but the threshold decreases with increase of $L$ (i.e., as the particles become less trapped).

We consider now the motion of a single potato particle, varying the perturbation amplitude. This will show us how the stochasticity arises. To see the orbit shape and topology more graphically, we plot intersections of the orbits with a poloidal cross section in conventional spatial co-ordinates. The results are presented in Fig. 12. We see that when the amplitude is small [Fig. 12 (a)], the perturbation does not change the orbit topology. Increase of the perturbation leads to resonance islands [Fig. 12 (b)], then secondary islands [Fig. 12 (c)], and, finally, stochasticity of the particle motion [Fig. 12 (d)].

To determine the dependence of the stochasticity threshold on the particle energy and tokamak parameters, we analyse the similarity properties of the following equations, which describe the guiding-center motion in a tokamak with concentric elliptic flux surfaces and $q \approx 1$ in the lowest order in the inverse aspect ratio:

$$
\dot{x}=-\frac{v_{\|} z}{k R_{0}}-\frac{v_{\|}}{B_{0} R_{0}} \frac{\partial \Psi}{\partial z}-\frac{c}{B_{0}} \frac{\partial \Phi}{\partial z},
$$




$$
\begin{gathered}
\dot{z}=\frac{k v_{\|} x}{R_{0}}-v_{\|} \frac{\partial}{\partial x}\left(\frac{v_{\|}}{\omega_{B}}\right)+\frac{v_{\|}}{B_{0} R_{0}} \frac{\partial \Psi}{\partial x}+\frac{c}{B_{0}} \frac{\partial \Phi}{\partial x}, \\
\dot{\varphi}=\frac{v_{\|}}{R_{0}}, \\
\dot{\mathcal{E}}=-e \frac{\partial \Phi}{\partial z} v_{\|} \frac{\partial}{\partial x}\left(\frac{v_{\|}}{\omega_{B}}\right)
\end{gathered}
$$

where $x=R-R_{0}, R$ is the distance to the axis of symmetry of the torus, $z$ is the vertical coordinate. Then we proceed to dimensionless variables $\tilde{x}=x /\left(R_{0} \nu\right), \tilde{z}=z /\left(k R_{0} \nu\right)[25,13]$ and take advantage of the fact that $v_{\|}=v \nu^{1 / 2} Y$, where $Y=Y(L, \tilde{x})$, which means that $v_{\|}$depends on the Larmor radius, $\rho$, as $v_{\|} \propto \rho^{4 / 3}$ at constant $L$. Assuming that the radial dependence of the perturbation is $X \propto r^{l}$, we find that Eqs. (25)-(27) become invariant to $\rho$ if we take $\Phi \propto \rho^{(8-2 l) / 3}, \Psi \propto \rho^{(4-2 l) / 3}$, but Eq. (28) does not. Hence, motion of particles of different energies is to be similar if they are launched with the same initial $\tilde{x}, \tilde{z}, \varphi$, and $L$. This similarity may be broken for electrostatic perturbations as they do not conserve the particle energy. But it can be expected to be valid for motion in a single resonance island as the energy change during such motion is small. As the stochasticity onset corresponds to overlap of separate islands, the similarity is likely to hold for the stochasticity threshold. Thus, assuming that the radial dependence of the $m=n=1$ mode is between $X \propto r$ (near the magnetic axis) and $X \propto r^{0}$ (at larger radii), we find for the threshold amplitude:

$$
\Psi_{\text {crit }} \propto \rho^{\gamma}, \quad \Phi_{\text {crit }} \propto \rho^{4 / 3+\gamma}
$$

where $\gamma=2 / 3 \div 4 / 3$, the lower and upper limits corresponding to particles localized at $r \ll r_{\max }$ and $r \sim r_{\text {max }}$, respectively.

In the case when the magnetic perturbation is produced by a sawtooth crash in a JET-like tokamak, its amplitude corresponds to that in Fig. 11, which implies that motion of 1-MeV potatoes during the crash is stochastic. Calculations carried out for electrostatic perturbations with amplitudes corresponding to the crash show that such perturbations also lead to stochastic motion of $1-\mathrm{MeV}$ potatoes. Therefore, we conclude that the crash results in stochastic motion of potato particles with $\mathcal{E} \lesssim 1 \mathrm{MeV}$. On the other hand, as follows from Eq. (29), the motion of particles of higher energies may be regular. For instance, as numerical calculations have shown, the motion of $5-\mathrm{MeV}$ ions is regular. However, one can expect that the combined action of the dominant harmonic of the crash and harmonics with $n>1$ will decrease the stochasticity threshold. If so, the motion of SAPs with the energy $\mathcal{E} \gtrsim 5 \mathrm{MeV}$ will be stochastic, too. The result depends also on characteristics of the crash.

One of the consequences of the stochasticity produced by the $m=n=1$ harmonic and the motion around SARD is transformation of quasi-stagnation particles into potatoes and largeorbit-width bananas. This implies that the mentioned mechanisms lead to expulsion of particles localized in the region with $J_{\theta} \rightarrow 0$. Therefore, they may contribute to disappearance of the hot spot during the sawtooth crash in the experiment on JET described in Subsec. IV D. Estimates show that the size of SARD of the hot spot ions is sufficiently large to be of importance. However, it is not clear whether the decrease/increase rate of the electromagnetic perturbation during the 
crash is sufficient to result in the particle displacement about the SARD width. The stochasticity induced by the $m=n=1$ mode may be an important factor affecting the hot spot ions provided that the crash amplitude and duration are sufficient for the stochasticity to manifest itself. A more detailed analysis is required to determine which of the described mechanisms dominate in the real experiment [10] and in the numerical experiment whose results are presented in Fig. 10.

\section{ESCAPING ALPHA PARTICLES IN TFTR}

Sawtooth crashes mainly redistribute fast ions within the plasma. But a small fraction of these ions can be expelled to the wall.

Energetic alpha particles escaping because of the crash were observed in TFTR DT experiments. Most of data came from the 1.4-MA shots at relatively low neutral beam injection power. Observations of the alpha loss were made using the lost alpha scintillation detectors located $90^{\circ}$, $60^{\circ}, 45^{\circ}$, and $20^{\circ}$ below the outer midplane (in the ion $\nabla B$-drift direction). It turned out that the alpha flux to the wall is strongly inhomogeneous, having sharp maxima at the $20^{\circ}$ and $90^{\circ}$ detectors and being on the noise level at the $45^{\circ}$ detector.

This experimental fact indicates that different physical mechanisms are responsible for the escape of alphas to the wall at $|\vartheta|>45^{\circ}$ and near the equatorial plane of the torus. A theory and numerical simulations in Refs. $[27,33]$ reveal these mechanisms.

In particular, a numerical simulation with the code OFSEF showed that only a narrow group of circulating particles can be expelled to the wall. These particles are marginally circulating and located sufficiently close to the magnetic axis before a crash. The process of the particle escape can be divided into two stages. First, interaction of the particles with the MHD perturbation, which expels the particle from the plasma core. This process is terminated by transformation of the circulating particle into a marginally trapped one, accompanied by a sharp change of the orbit width. After the transformation occurs, the particle can be lost within one bounce, see Fig. 13. Therefore, this process can be referred to as "crash-induced prompt loss". Only particles with sufficiently high energy, $\mathcal{E} \geq \mathcal{E}_{\text {min }}$ can be lost, and the lost particles reach the wall at $\vartheta_{\min } \leq|\vartheta| \leq \vartheta_{\max }$ (either below or above the midplane of the torus, depending on the direction of the toroidal magnetic field).

Trapped particles escape from the plasma because of the "crash-induced stochastic diffusion", i.e., they are transported by the crash to region of stochastic diffusion and then are lost for many bounce periods. These particles are detected mainly by the $20^{\circ}$ detector. This was found with using the Hamiltonian guiding-center code ORBIT [28,34] and the dependence of $\vartheta_{\tau}$ on $\lambda$ given in Fig. 14, where $\vartheta_{\tau}$ is the angle at which the orbit of an escaping ion is tangent to the wall. Calculations with ORBIT showed that when the alpha source vanishes outside the sawtooth

mixing radius (which models the crash-induced source), the dominant fraction of the escaping particles consists of moderately trapped particle with the energy close to $3.5 \mathrm{MeV}$ and $\lambda \gtrsim 1$. Therefore, according to Fig. 14, the crash-induced stochastic diffusion leads to alpha wall load at $|\vartheta|<30^{\circ}$. It also follows from Fig. 14 that the particles with $\lambda \sim 0.8$, which is typical for the particles escaping due to the orbit transformation, reach the wall at $|\vartheta|>30^{\circ}$. Note that the dependence of $\vartheta_{\tau}$ on $\lambda$ shown in Fig. 14 is rather general, being almost independent on the specific mechanism leading to the particle loss. 
In order to describe the poloidal distribution of the alpha flux to the wall for $|\vartheta| \geq 30^{\circ}$, a theory based on the assumption that the crash duration essentially exceeds the particle transit time / bounce period was suggested [33]. There is reasonable agreement between predictions of this theory and experimental data, see Fig. 15.

Thus, the dominant physical mechanisms responsible for the alpha particle escape are the crash-induced prompt loss leading to wall load mainly near the chamber bottom and the crashinduced stochastic diffusion resulting in wall load near the midplane of the torus. The first mechanism leads to the escape of particles which were circulating before the crash, whereas the second one affects mainly trapped particles.

\section{SUMMARY AND CONCLUSIONS}

Our knowledge on the sawtooth-induced transport of fast ions can be summarized as follows. When the particle energy is less than $\mathcal{E}_{\text {crit }}$, all particles are strongly redistributed by the crash. The behaviour of particles with $\mathcal{E}>\mathcal{E}_{\text {crit }}$ essentially depends on their pitch angles. When $\mathcal{E}>$ $\mathcal{E}_{\text {crit }}$, circulating particles are strongly redistributed, whereas only resonant trapped particles are essentially affected. There are two kinds of the resonances. First, resonances of the unperturbed particle motion with the electromagnetic field of the crash. Such resonances provide particle redistribution due to interaction with $n>1$ harmonics, which appear at the non-linear stage of the crash if it results from the $m=n=1$ instability. The exception is a group of particles with very weak toroidal precession, which interacts with any harmonic. Second, resonances which arise for the $n=1$ mode as a consequence of the finite amplitude of the perturbation. They are due to the influence of the perturbation on the particle motion, which modifies the frequencies of the particle motion and the spectral composition of the perturbation in the particle reference frame.

The particles with $\mathcal{E}<\mathcal{E}_{\text {crit }}$ are redistributed within the sawtooth mixing region. The resonant particles with $\mathcal{E}>\mathcal{E}_{\text {crit }}$ are redistributed near the resonant drift surfaces. Their displacement is determined by the width of superbanana orbits unless the resonance islands overlap, leading to stochasticity. However, the stochasticity can manifest itself only when the characteristic time of the stochastic diffusion is less than the crash duration. Note that the stochasticity can arise even in the presence of a monochromatic $m=n=1$ perturbation, its amplitude threshold being the smallest for potatoes.

There is an additional mechanism of redistribution of particles with potato and quasistagnation orbits, which results from combined action of the particle motion along perturbed orbits and quick switching on/off the perturbation.

It is convenient to present the dependence of the behaviour of different groups of energetic ions during sawtooth oscillations on the parameters of the ions and the device in terms of the normalized particle energy and crash duration, see Fig. 16. Using Fig. 16, one can not only understand the different effect of sawtooth crashes on fast ions in present-day experiments but also make certain predictions. In particular, one can see that alpha particles will be strongly redistributed by crashes in large machines (in, e.g., ITER).

Finally, we note that the study of the sawtooth-induced redistribution of fast ions can provide information on the physics of the sawtooth crash itself. In particular, it is hard to expect that 
stochasticity of magnetic field lines or turbulence, which are often believed to be the main reason of the sawtooth crash, can lead to creation of hollow post-crash profiles of fast ions. In addition, experimental evidence of the existence of $\mathcal{E}_{\text {crit }}$ given by Eq. (5) supports the assumption that the $m=n=1$ mode is the main driving force of the redistribution. If higher modes were dominant, this would lead to much lower critical energy. Thus, available experimental information and our studies are in favour of the crash models that attribute the crash to development of the $m=n=1$ instability [14-16] or, at least, show that the $m=n=1$ instability plays the main role in the redistribution.

Acknowledgments. The research described in this publication was made possible in part by the Award No. UP2-290 of the Government of Ukraine and the U.S. Civilian Research \& Development Foundation for the Independent States of the Former Soviet Union. The authors thank V.S.Marchenko for useful discussions. 


\section{REFERENCES}

[1] Von Goeler, S., Stodiek, W., Sauthoff N., Phys. Rev. Lett. 33 (1974) 1201.

[2] Sadler, G.J., et al., Fusion Technol. 18 (1990) 556.

[3] Marcus, F.B., et al., Plasma Phys. Control. Fusion 31 (1991) 277.

[4] Marcus, F.B., et al., Nucl. Fusion 33 (1993) 1325.

[5] Stratton, R.C., et al., Nucl. Fusion 36 (1996) 1586.

[6] Petrov M.P., et al., Nucl. Fusion 35 (1995) 1437.

[7] Petrov, M.P., et al., in Fusion Energy 1996 (Proc. 16th IAEA Conf. Montreal, 1996), Vol. 1, IAEA, Vienna (1997) 261.

[8] Gorelenkov, N.N., et al., Nucl. Fusion 37 (1997) 1053.

[9] Medley, S.S., et al., Nucl. Fusion 38 (1998) 1283.

[10] Jarvis, O.N., et al., Nucl. Fusion 36 (1996) 1513.

[11] Kolesnichenko, Ya.I., Yakovenko, Yu.V., in Collection of papers presented at the IV IAEA Tech. Comm. Mtg on Alpha Particles in Fusion Research (Princeton, April 1995); Nucl. Fusion 36 (1996) 159.

[12] Kolesnichenko, Ya.I., Lutsenko, V.V., Yakovenko, Yu.V., White, R.B., Yi Zhao, in Alpha Particles in Fusion Research (Proc. 5th IAEA Tech. Comm. Mtg Abingdon, 1997), Joint European Torus, Abingdon (1997) 181.

[13] Kolesnichenko, Ya.I., Lutsenko, V.V., White, R.B., Yakovenko, Yu.V., Phys. Plasmas 5 (1998) 2963.

[14] Kadomtsev, B.B., Fiz. Plazmy 1 (1975) 710 [in Russian]; Sov. J. Plasma Phys. 1 (1976) 389.

[15] Kolesnichenko, Ya.I., Yakovenko Yu.V., Anderson, D., Lisak, M., Wising, F., Phys. Rev. Lett. 68 (1992) 3881.

[16] Biskamp, D., Drake, J.F., in Plasma Physics and Controlled Nuclear Fusion Research (Proc. 15th IAEA Conf. Seville, 1994), Vol. 3, IAEA, Vienna (1995) 261.

[17] Kolesnichenko, Ya.I., Yakovenko, Yu.V., Nucl. Fusion 32 (1992) 449.

[18] Anderson, D., Kolesnichenko, Ya.I., Lisak, M., Wising, F., Yakovenko, Yu.V., Nucl. Fusion 34 (1994) 217.

[19] Anderson, D., Kolesnichenko, Ya.I., Lisak, M., Wising, F., Yakovenko, Yu.V., "Influence of sawtooth oscillations on fast ion spatial distribution", CTH-IEFT/PP-1992-22, Chalmers University of Technology, Gothenburg (1992).

[20] Yi Zhao, White, R.B., Phys. Plasmas 4 (1997) 1103.

[21] Kolesnichenko, Ya.I., Lutsenko, V.V., Yakovenko, Yu.V., Kamelander, G., Phys. Plasmas 4 (1997) 2544.

[22] Kolesnichenko, Ya.I., Lutsenko, V.V., Yakovenko, Yu.V., Kamelander, G., in Controlled Fusion and Plasma Physics (Proc. 23nd Eur. Conf. Kiev, 1996), Vol. 20C, Part I, European Physical Society, Geneva (1996) 331.

[23] Kolesnichenko, Ya.I., Lutsenko, V.V., Yakovenko, Yu.V., Phys. Plasmas 5 (1998) 729.

[24] Galeev, A.A., Sagdeev, R.Z., in Problems of Plasma Theory, Vol. 7, Consultants Bureau, New York (1979) 257.

[25] Porcelli, F., Eriksson, L.-G., Berk, H.L., in Controlled Fusion and Plasma Physics (Proc. 21st Eur. Conf. Montpellier, 1994), Vol. 18C, Part 2, European Physical Society, Geneva (1994) 648. 
[26] Yakovenko, Yu.V., in 1998 International Congress on Plasma Physics combined with 25th European Physical Society Conference on Controlled Fusion and Plasma Physics (Praha, 1998), Vol. 22C, European Physical Society, Geneva (1998) 2133.

[27] Kolesnichenko, Ya.I., Lutsenko, V.V., White, R.B., Yakovenko, Yu.V., Zweben, S.J., "Behaviour of $\mathrm{MeV}$ ions in the presence of sawtooth oscillations in TFTR and JET", paper THP2/25 at 17th IAEA Fusion Energy Conference (Yokohama, 1998) (in press).

[28] White, R.B., Chance, M.S., Phys. Fluids 27 (1984) 2455.

[29] Kaufman, A.N., Phys. Fluids 15 (1972) 1063.

[30] Yakovenko, Yu.V., Kolesnichenko, Ya.I., Lutsenko, V.V., White, R.B., "Small-action resonance in Hamiltonian systems and redistribution of energetic ions in tokamaks", PPPL Rep. 3349, Princeton Plasma Physics Laboratory, Princeton (1999).

[31] Littlejohn, R.G., J. Math. Phys. 23 (1982) 742.

[32] Lichtenberg, A.J., Lieberman, M.A., Regular and stochastic motion, Springer-Verlag, Berlin, Heidelberg, and New York (1983).

[33] Kolesnichenko, Ya.I., Lutsenko, V.V., White, R.B., Yakovenko, Yu.V., Zweben, S.J., Phys. Plasmas 6 (1999) 1117.

[34] White, R.B., Phys. Fluids B 2 (1990) 845. 


\section{FIGURES}

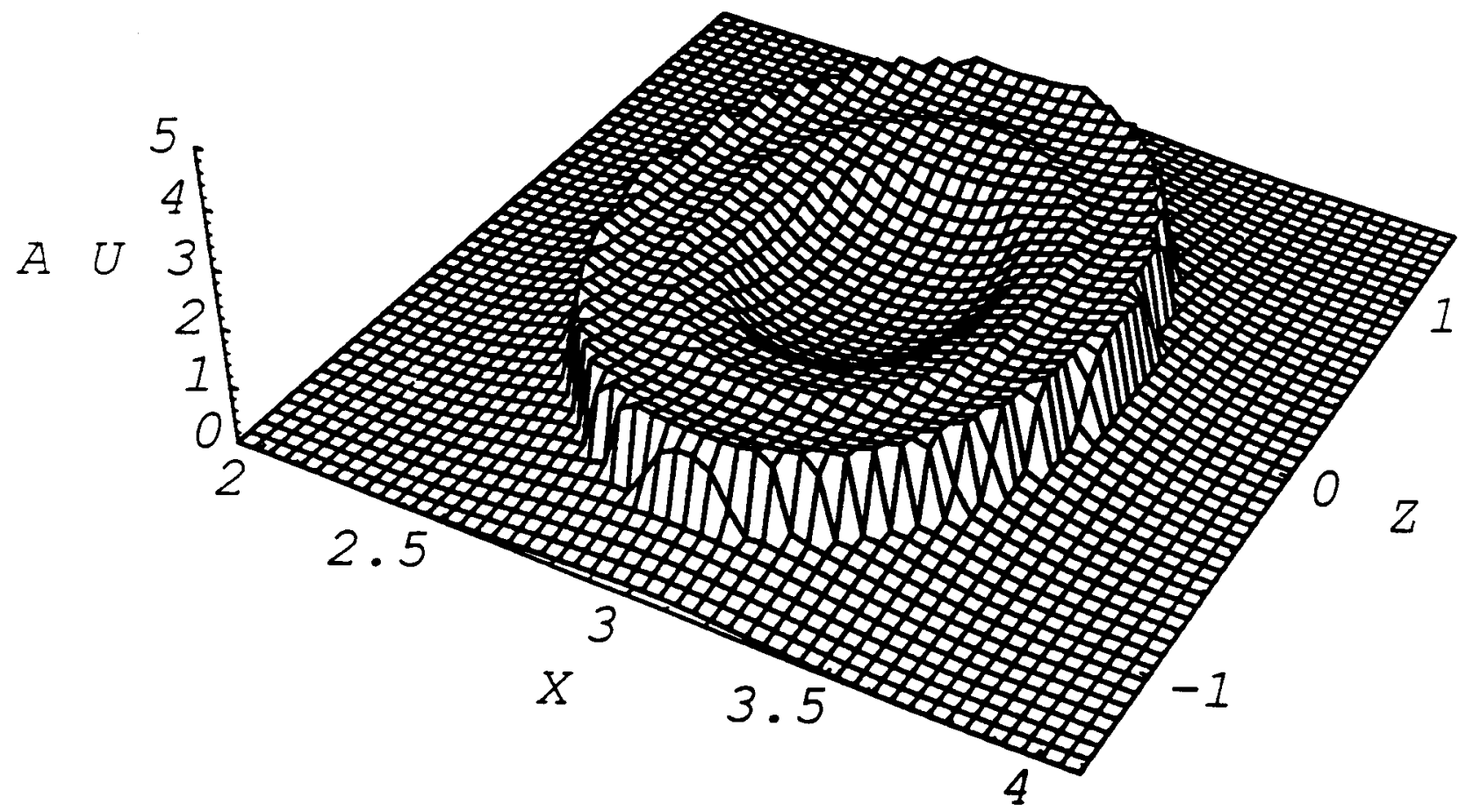

FIG. 1. The calculated post-crash profile of neutron emission in NBI experiment on JET, pulse \#20222 [19]. Approximation of flux-surface-attached particles and the crash model of Ref. [15] were used. 


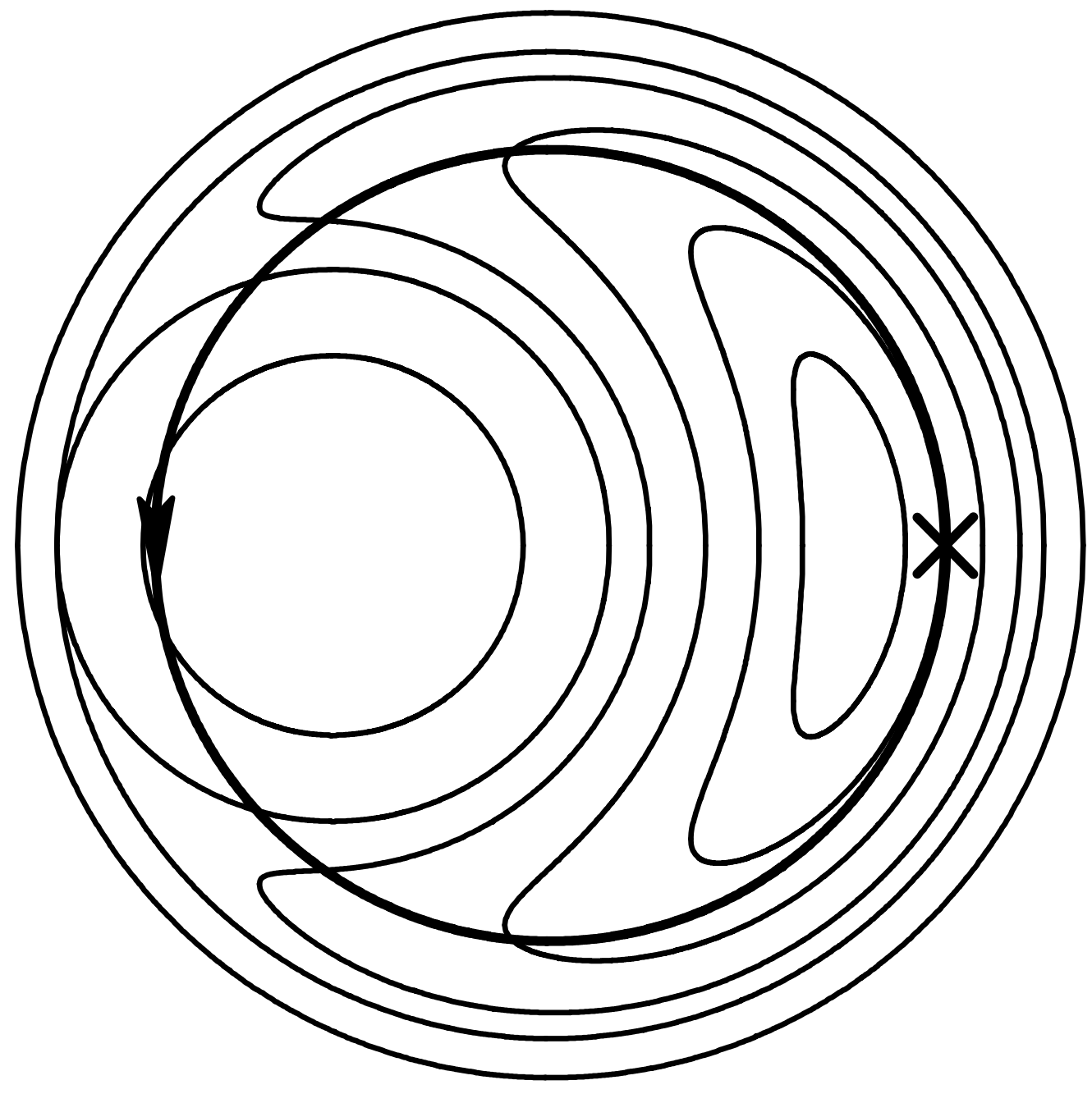

FIG. 2. Sketch of bounce-averaged orbits of particles with $\tau_{p} \ll \tau_{c r}$ (bold line) and $\tau_{p} \gg \tau_{c r}$ (cross) for a time interval $\Delta t \ll \tau_{c r}$. Plain lines are partly reconnected magnetic flux surfaces. The particle with $\tau_{p} \ll \tau_{c r}$ moves across the flux surfaces at $r \approx$ const, whereas the particle with $\tau_{p} \gg \tau_{c r}$ is attached to a flux surface and, thus, moves together with it. 


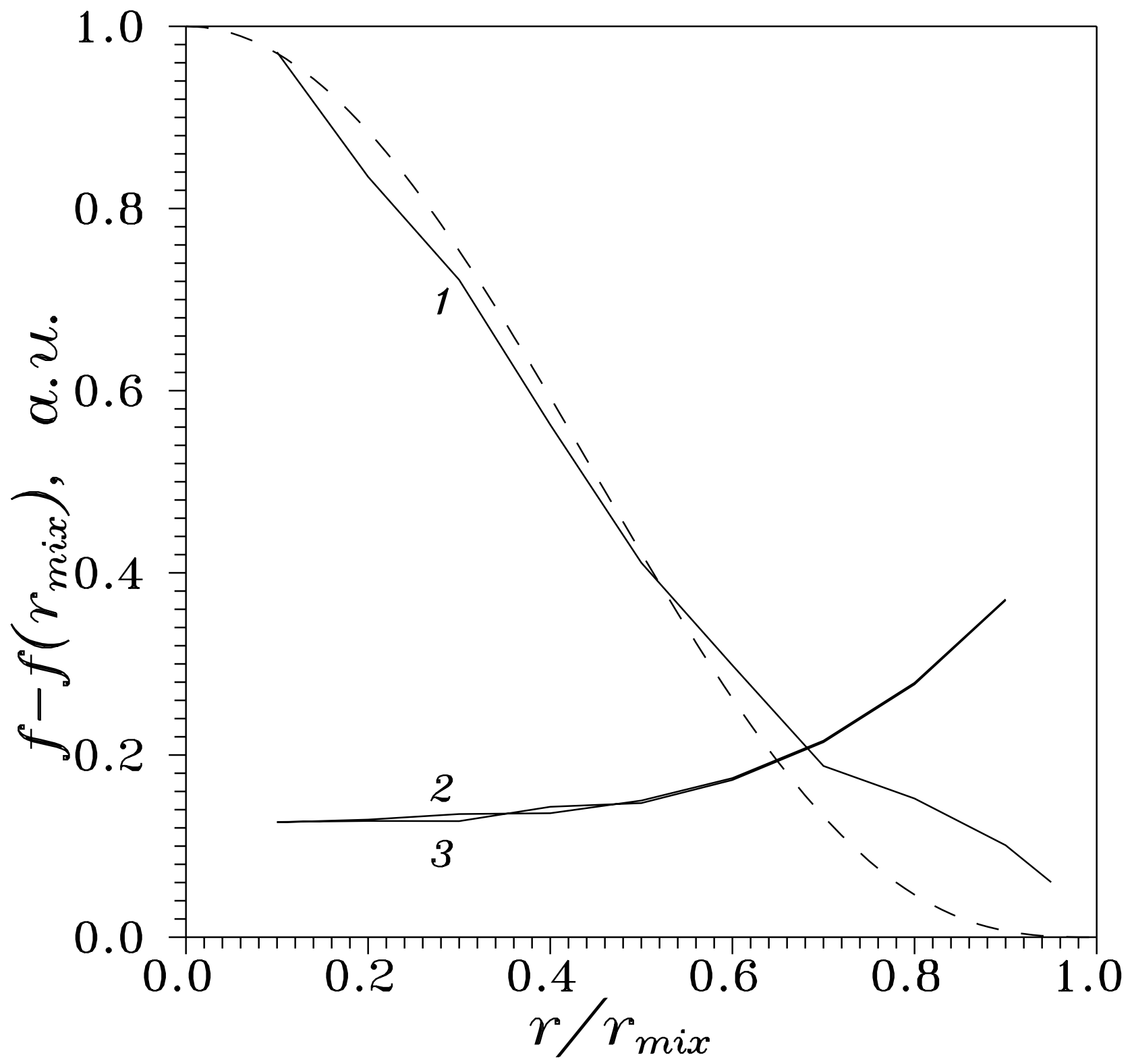

FIG. 3. Crash-induced redistribution of trapped and circulating $\alpha$-particles with $\mathcal{E}=2.8$ $\mathrm{MeV}$ in JET. Notations: dashed line, pre-crash distribution; solid lines, post-crash distributions. 1, trapped particles with $\lambda=1 ; 2$ and 3, co- and counter-circulating particles with $\lambda=0.375$; $\lambda=\mu B_{0} / \mathcal{E}, \mu$ is the magnetic moment. 


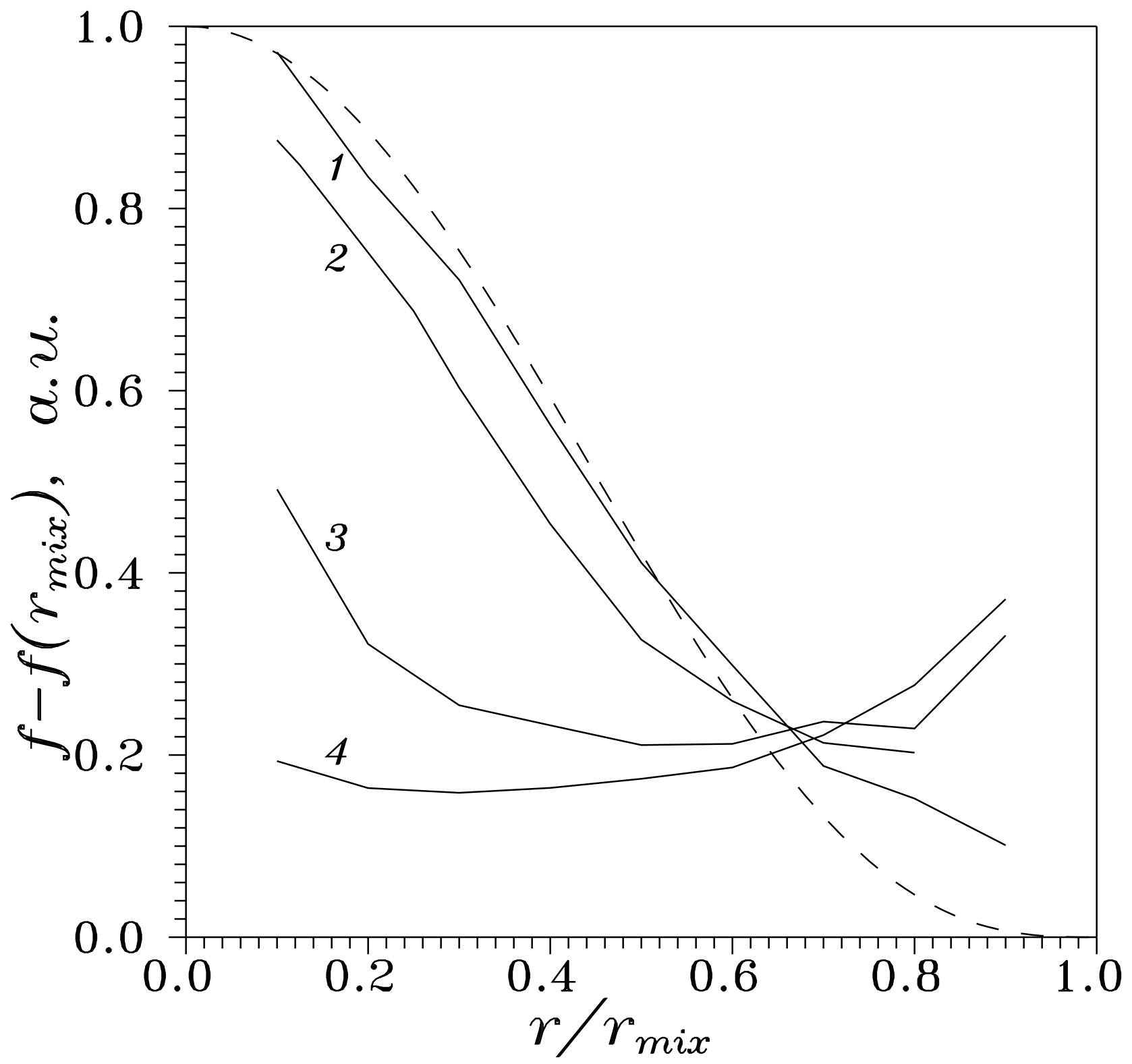

FIG. 4. Crash-induced redistribution of trapped $\alpha$-particles with $\lambda=1$ and various energies in JET. Notations: $1, \mathcal{E}=2.8 \mathrm{MeV} ; 2, \mathcal{E}=1.4 \mathrm{MeV} ; 3, \mathcal{E}=0.56 \mathrm{MeV} ; 4, \mathcal{E}=0.28 \mathrm{MeV}$; other notations are the same as in Fig. 3. 


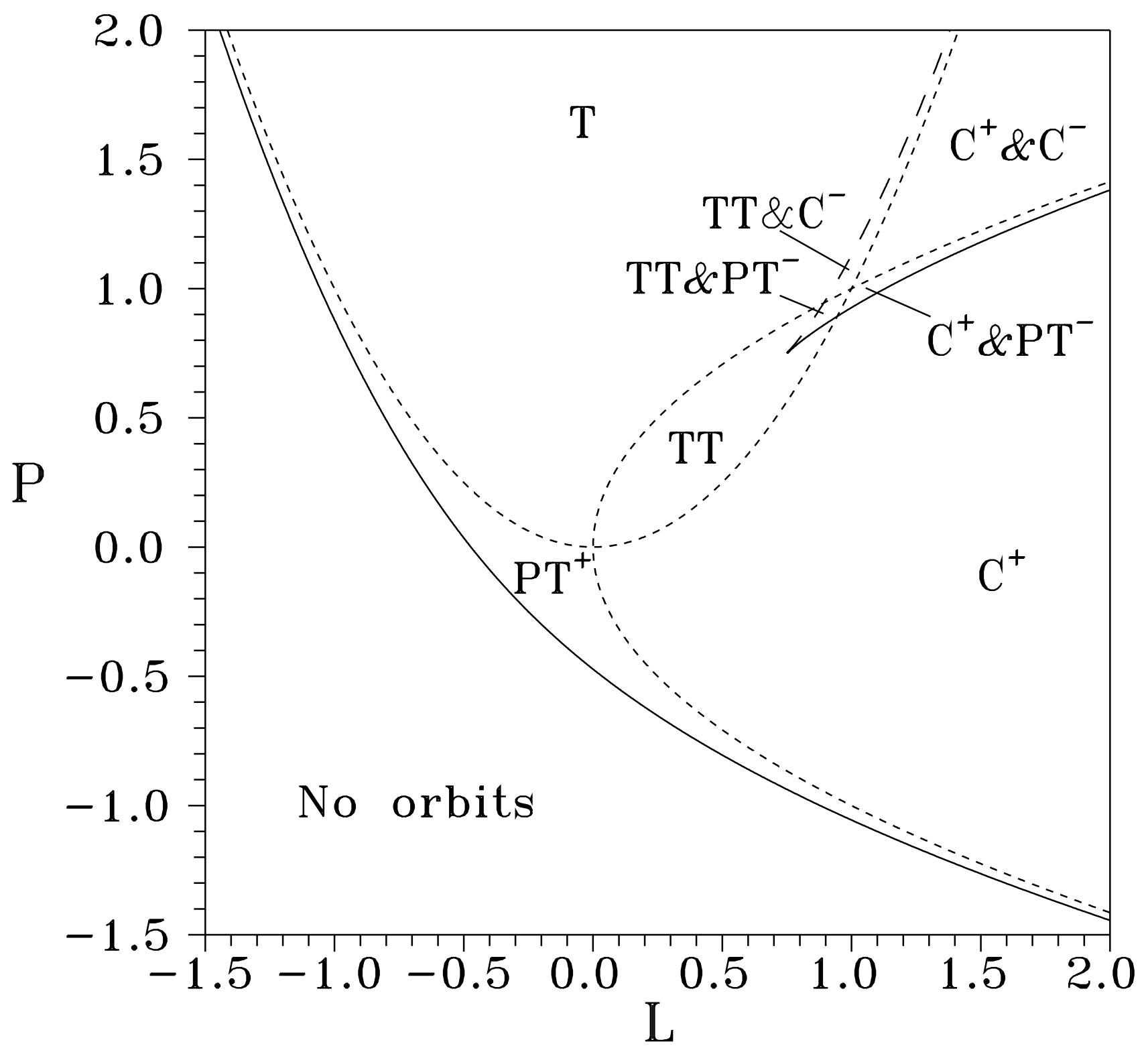

FIG. 5. Classification of particle orbits. Notations: C, circulating; PT, poloidally trapped; TT, toroidally trapped; T, trapped (both poloidally and toroidally); superscripts '+' and '-' refer to $\operatorname{sgn} v_{\|}$; solid lines, loci of stagnation orbits; dashed line, locus of orbits with X-points; $P \gg 1$ corresponds to standard orbits with $\Delta r_{b} \ll r$. 


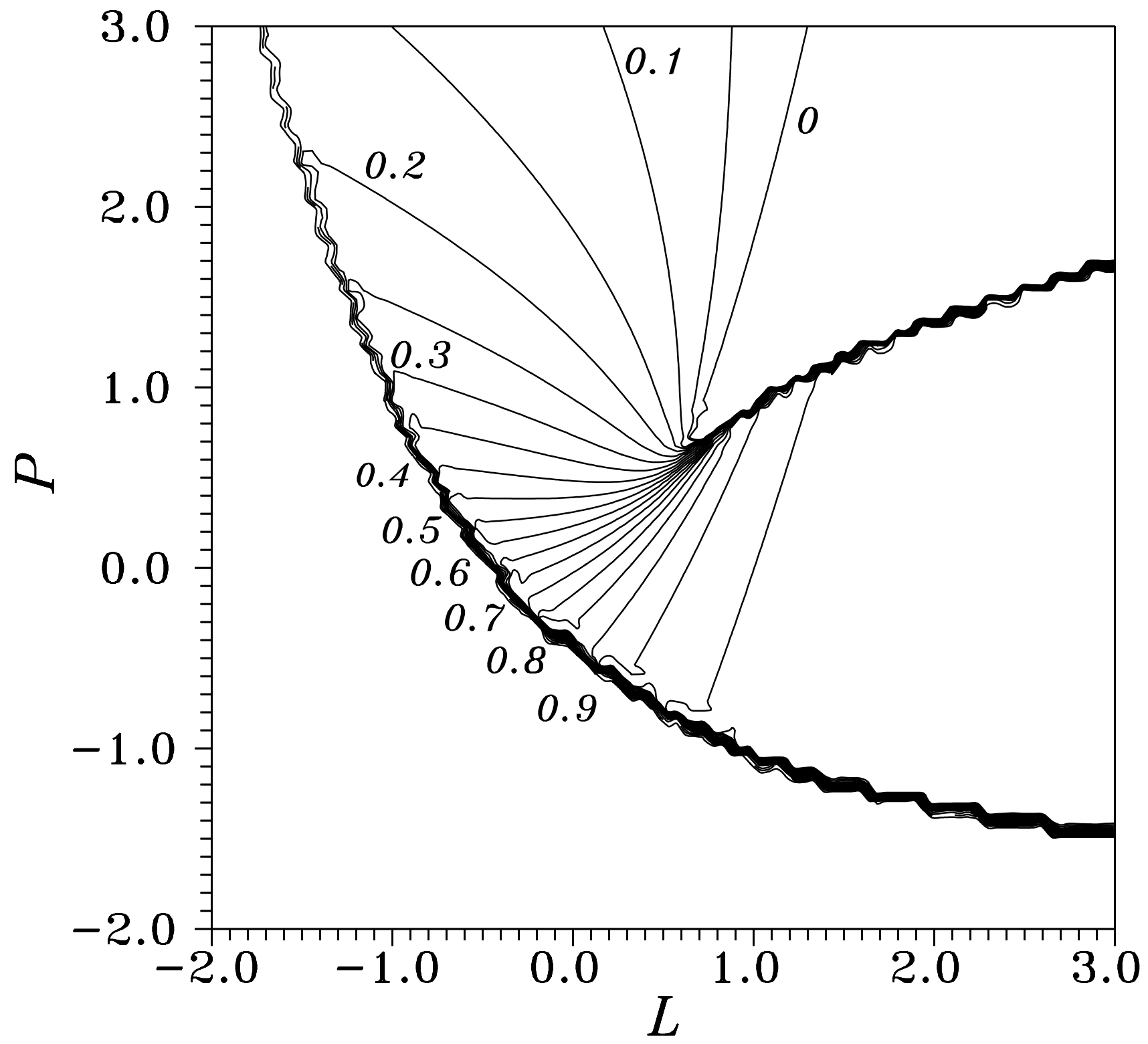

FIG. 6. Level contours of $\omega_{\varphi} /\left(q \omega_{b}\right)$ for $\nu=0.11$. 


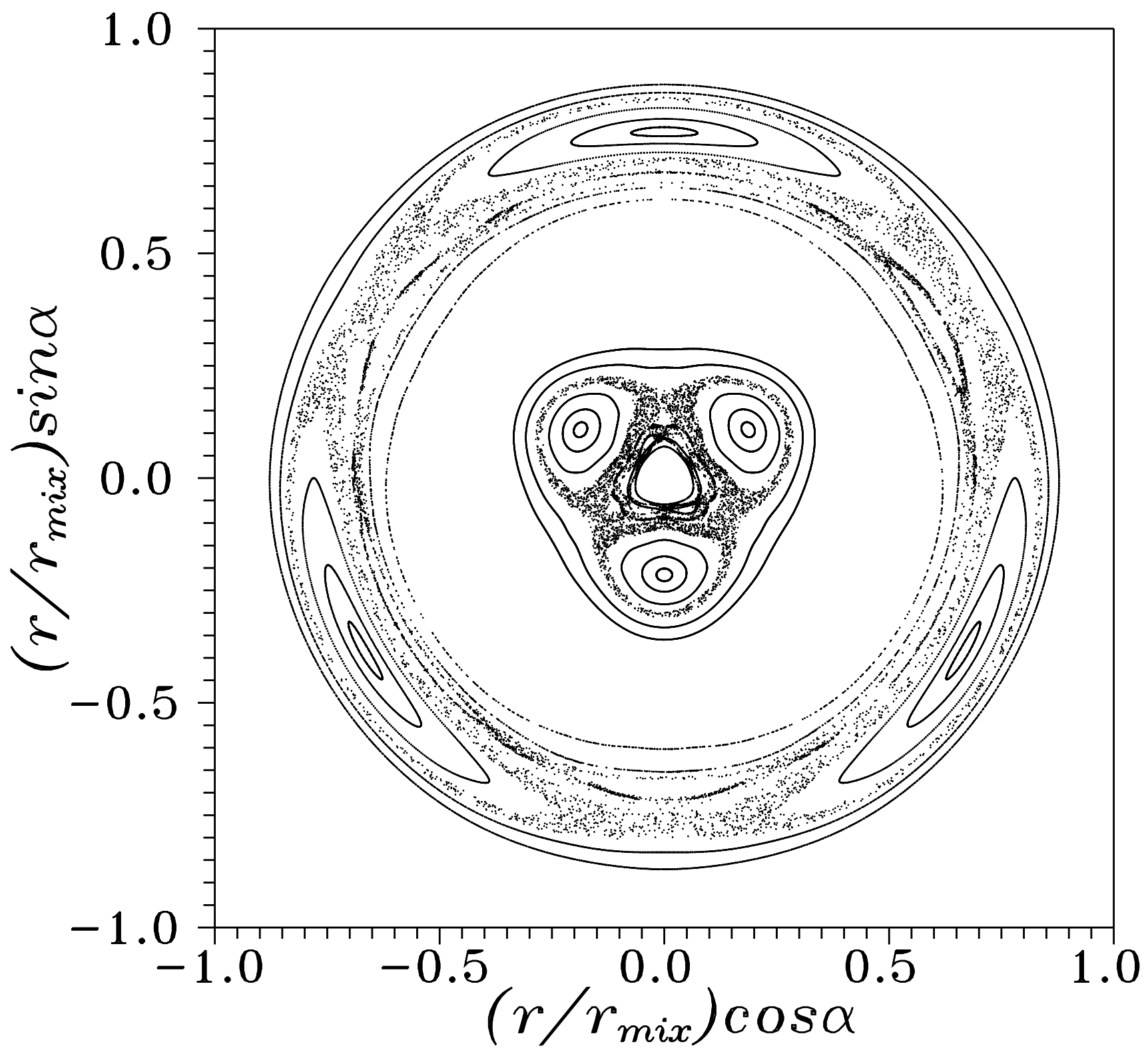

FIG. 7. Poincaré map showing several trapped (superbanana) and passing orbits and a stochastic region in the presence of the perturbation with $\Phi_{\max }=0.15 k B_{0} r_{\text {mix }}^{2} /\left(c \tau_{c r}\right)$. Used parameters: $\mathcal{E}=1.4 \mathrm{MeV}, \lambda=1.007, B_{0}=3.5 \mathrm{~T}, R_{0}=3.0 \mathrm{~m}, \tau_{c r}=10^{-4} \mathrm{~s}, r_{\text {mix }}=0.4 \mathrm{~m}$, $k=1.5$. 


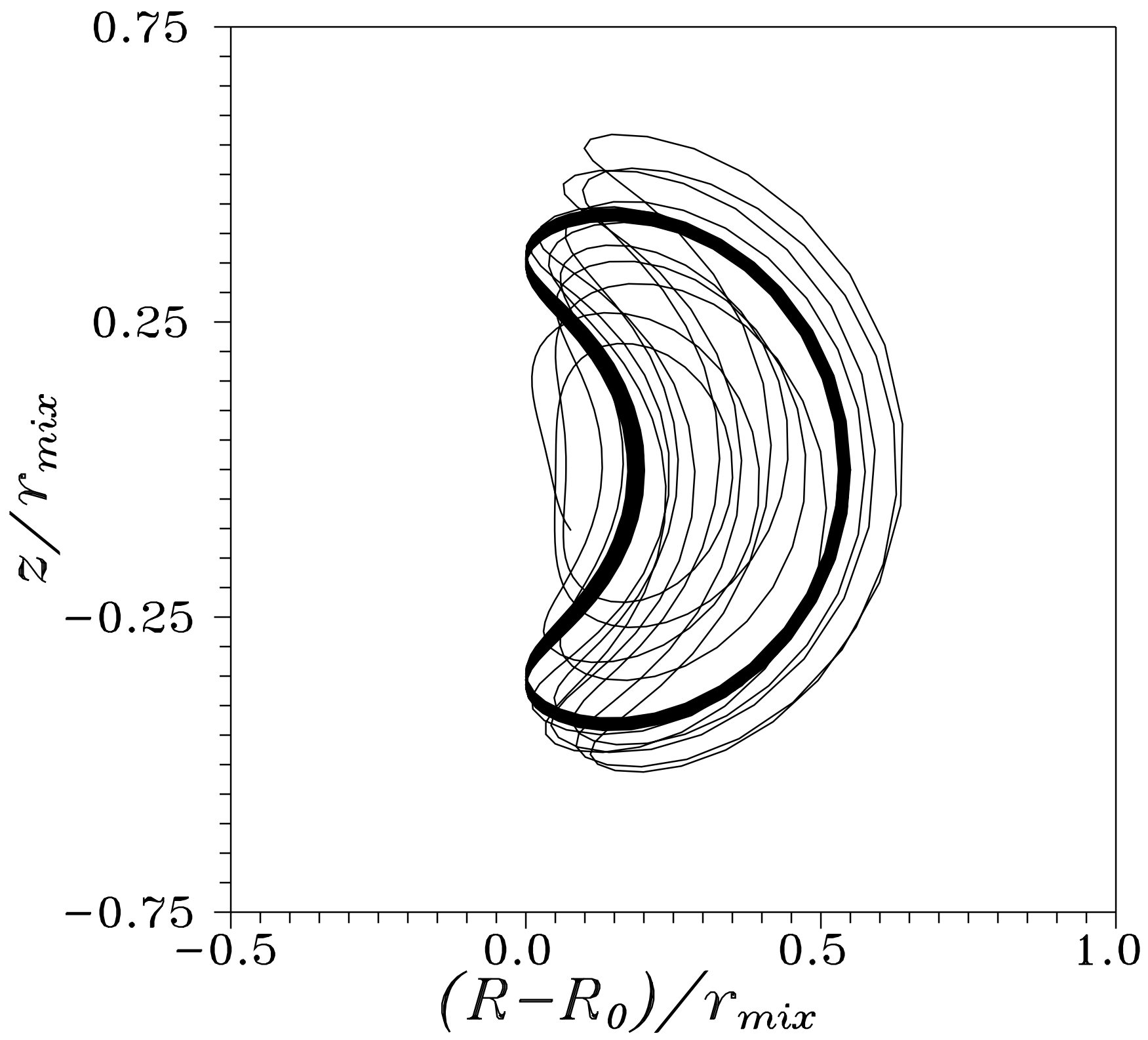

FIG. 8. The effect of a sawtooth crash on a typical fast ion with $\mathcal{E}=2 \mathrm{MeV}$ in JET. Bold line, pre-crash ion orbit; thin line, the orbit during the crash. The crash duration is $10^{-4} \mathrm{~s}$; the sawtooth mixing radius, $80 \mathrm{~cm}$. 


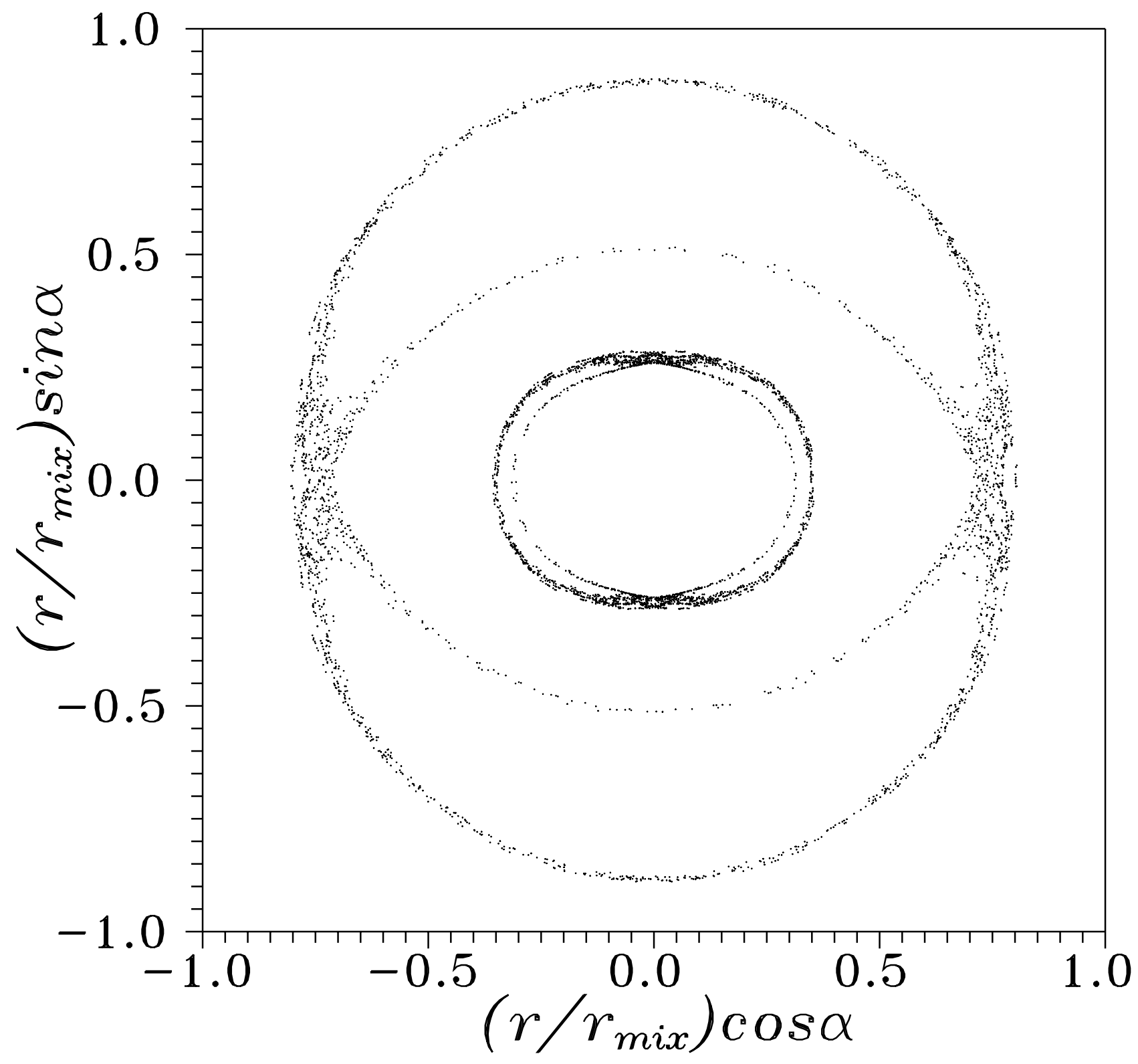

FIG. 9. Poincaré maps of motion of an ion relevant to the hot spot [10] under an $m=n=2$ electrostatic perturbation of the form $\Phi=\Phi_{\max } f_{2}(r) \sin (2 \alpha)$, where $f_{2}(r)=4\left(r / r_{m i x}\right)^{2}\left[1-\left(r / r_{m i x}\right)^{2}\right]\left(\max f_{2}(r)=1\right), \Phi_{\max }=0.32 k B_{0} r_{\text {mix }}^{2} /\left(c \tau_{c r}\right)$. The used parameters are the same as in Fig. 7 . 


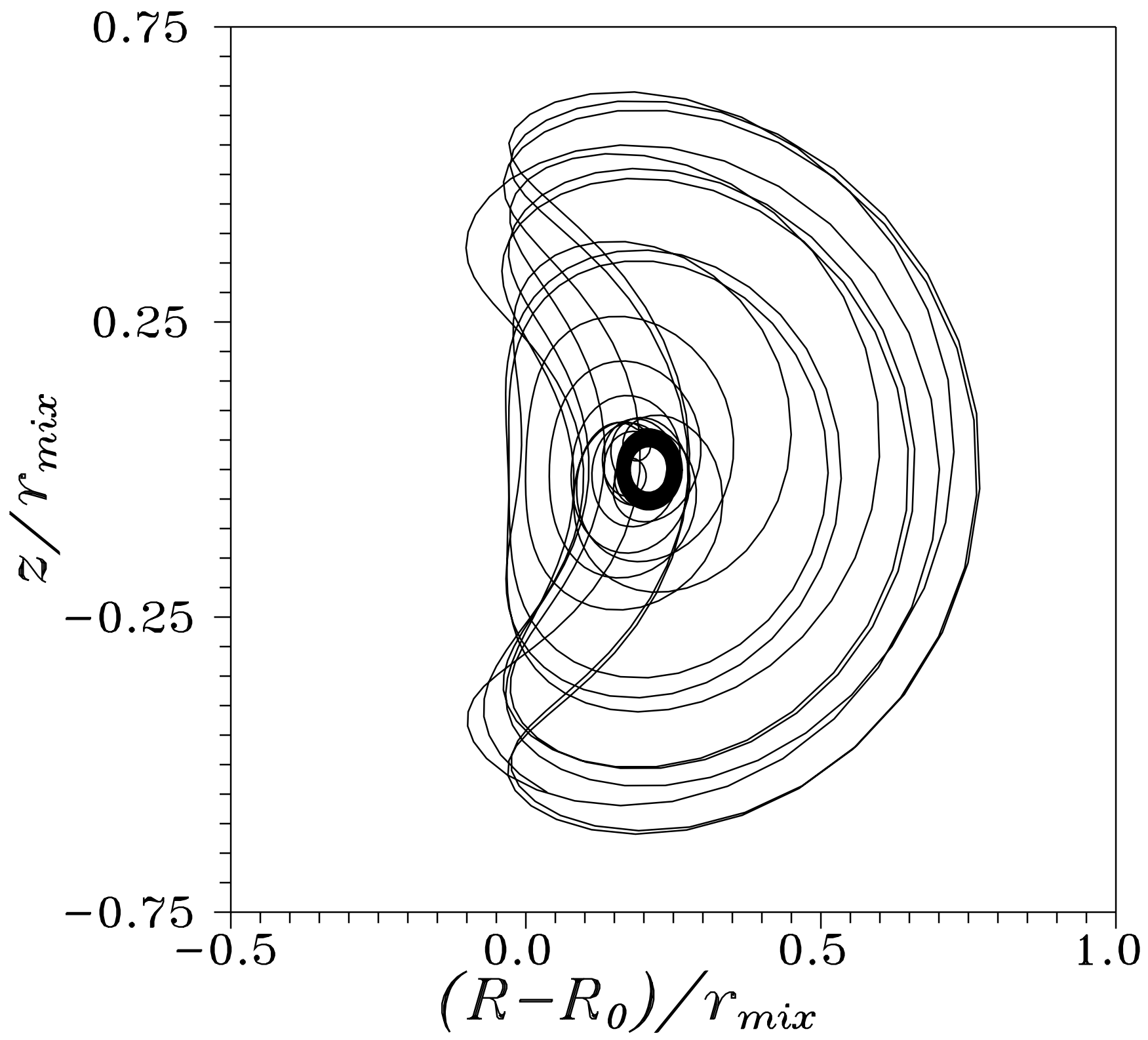

FIG. 10. The effect of a crash on a hot-spot ion with $\mathcal{E}=5 \mathrm{MeV}$. The crash parameters and notations are the same as in Fig. 8. 


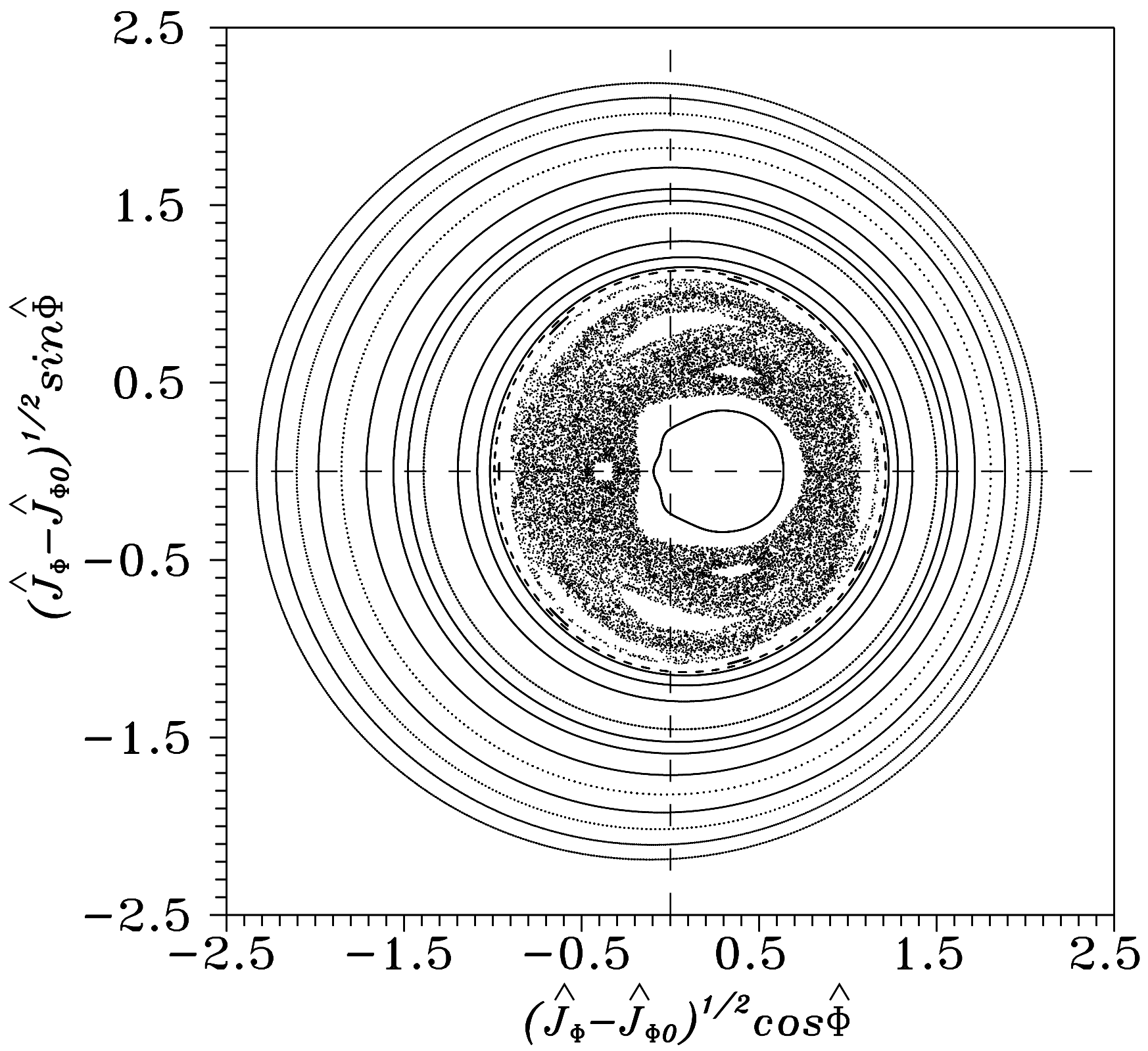

FIG. 11. The Poincaré map of $1-\mathrm{MeV}^{3} \mathrm{He}$ ions in JET in the presence of an $m=n=1$ perturbation with the normalized amplitude $\Psi_{\max }=0.05 k B_{0} r_{\max }^{2}$, where $B_{0}=3.5 \mathrm{~T}, k=1.2$, $r_{\max }=60 \mathrm{~cm}$. The points where particles cross the equatorial plane of the torus, moving downwards, are presented. 

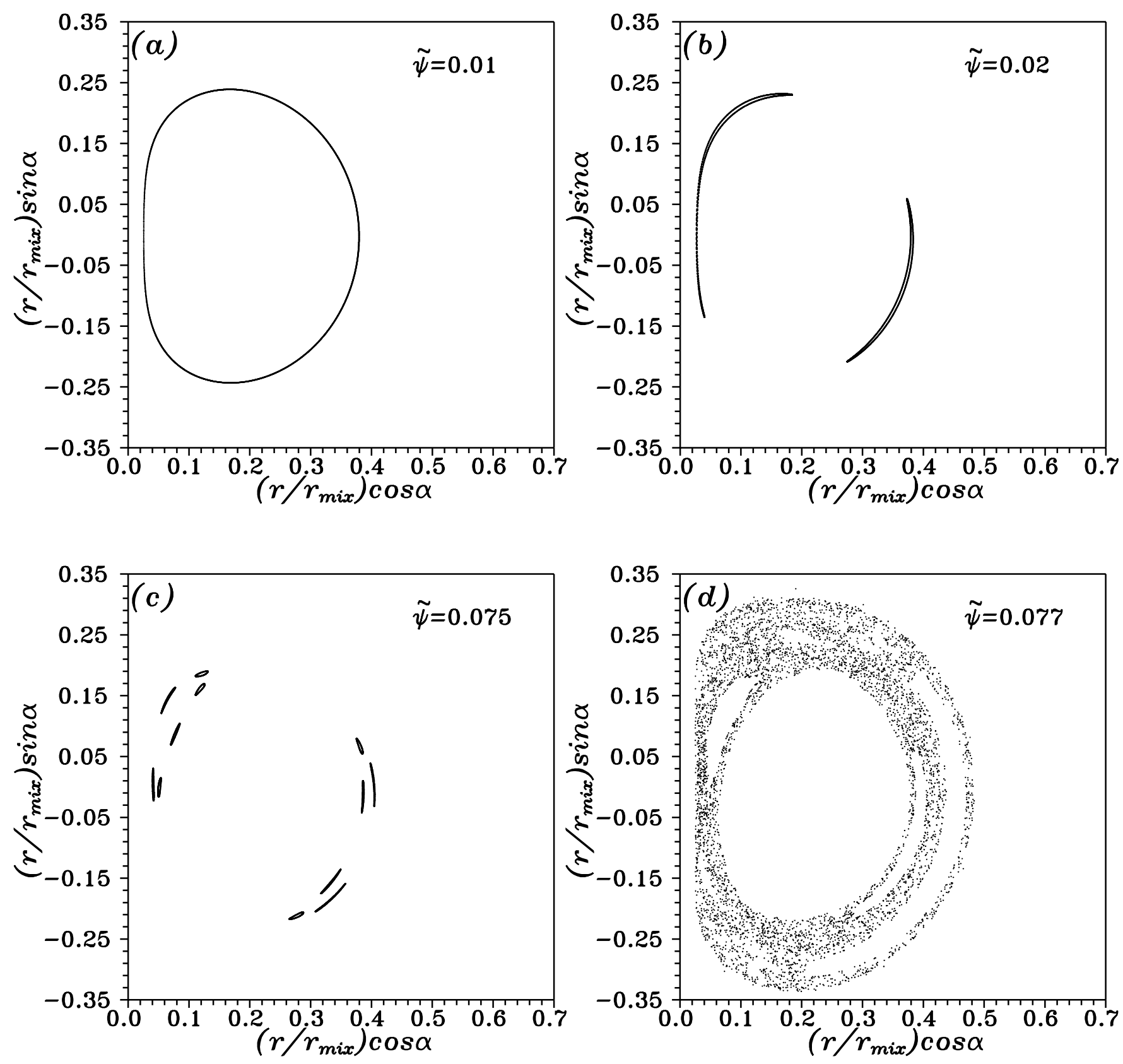

FIG. 12. Poincaré maps of the motion of a $1-\mathrm{MeV}{ }^{3} \mathrm{He}$ ion in JET under $m=n=1$ perturbations of the magnetic field of various amplitudes. The points where particles cross a poloidal plane of the torus are presented. $\tilde{\Psi}=2 \Psi_{\max } /\left(k B_{0} r_{\text {max }}^{2}\right)$. The used parameters are the same as in Fig. 11. 


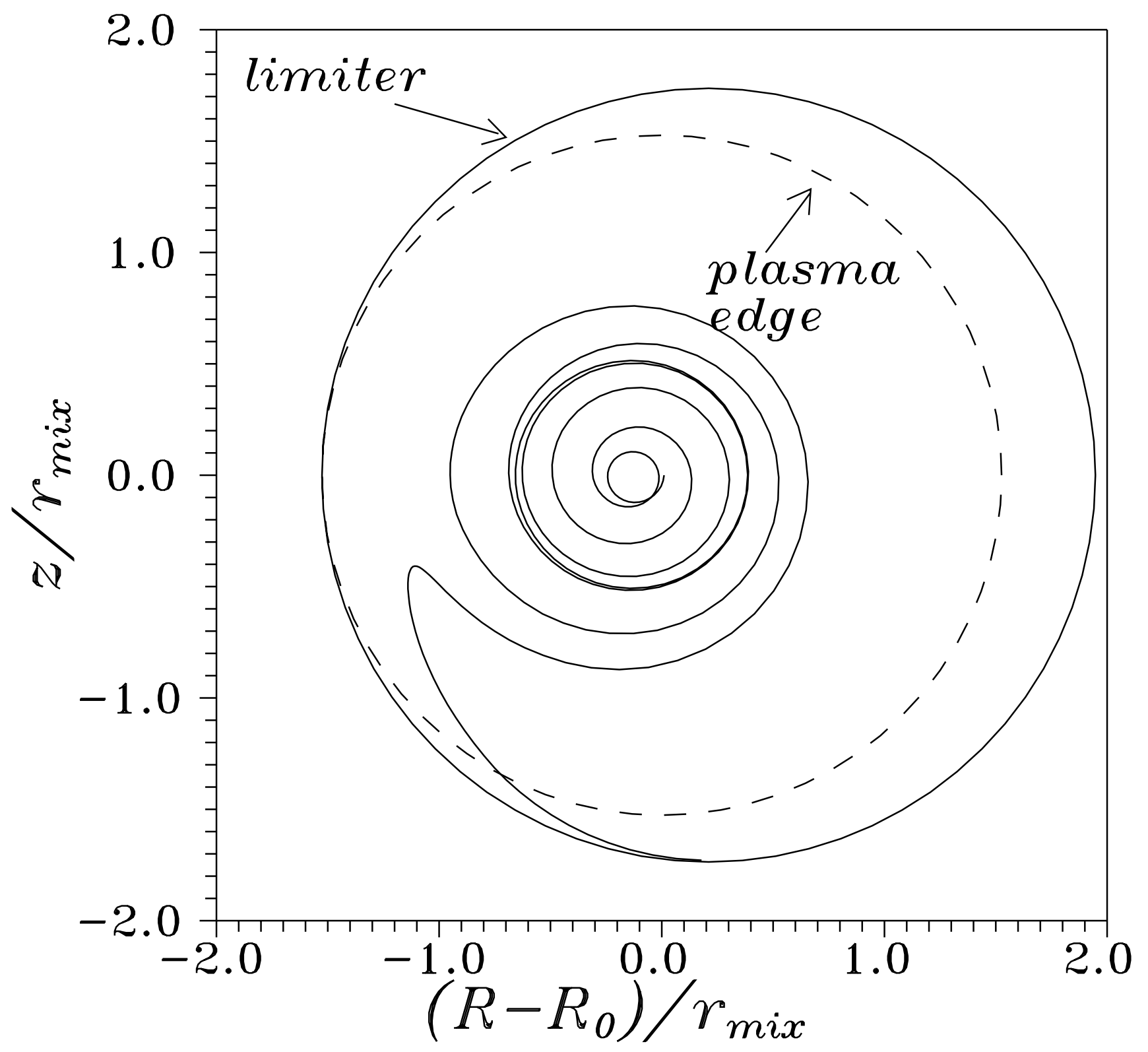

FIG. 13. Crash-induced escape of an alpha particle with $\lambda=0.75$ and $\mathcal{E}=2.5 \mathrm{MeV}$ in TFTR. 


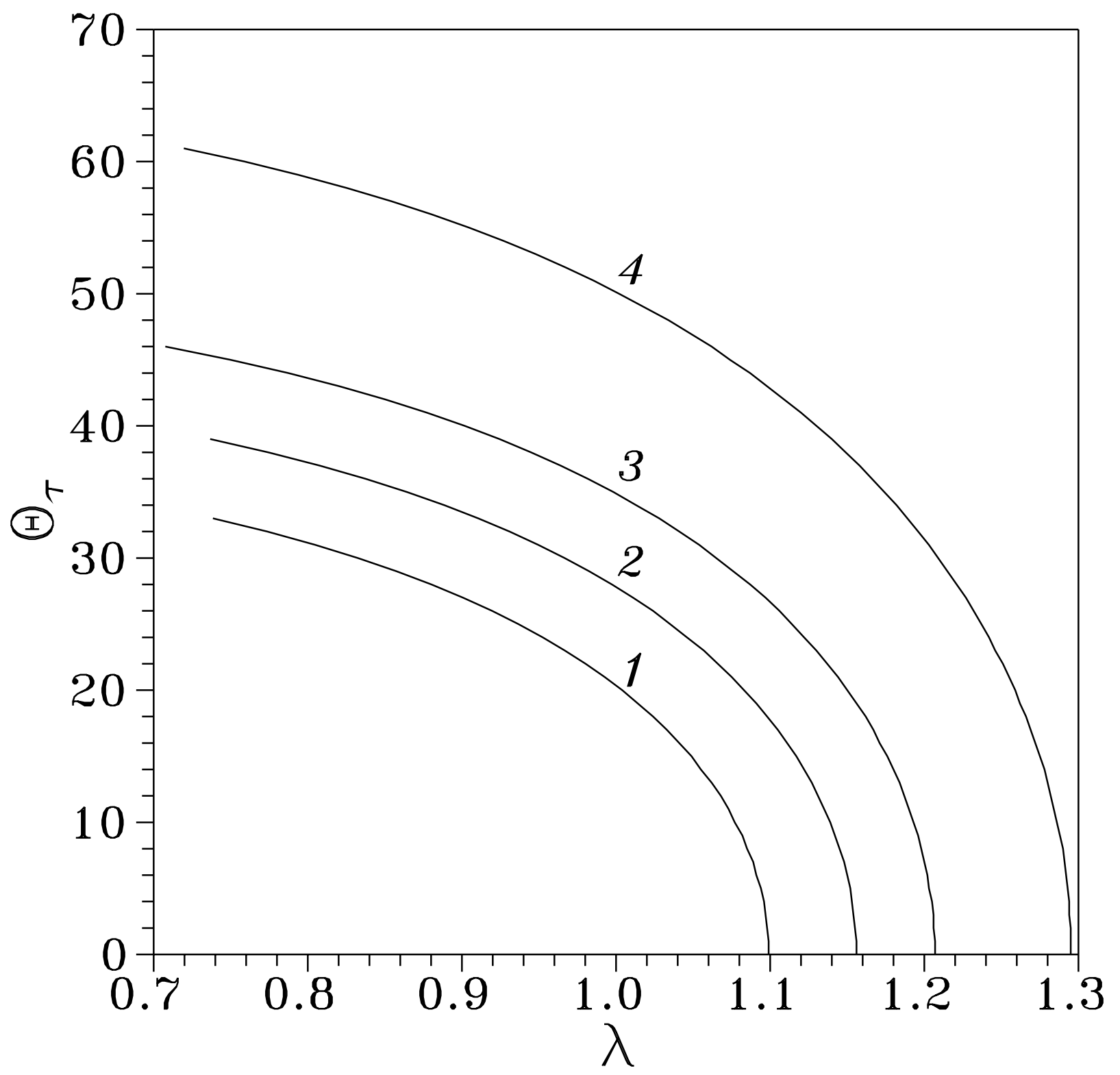

FIG. 14. $\vartheta_{\tau}$ vs $\lambda$ for various values of $\mathcal{E}$ in TFTR shot \#87530. 1, $\mathcal{E}=3.5 \mathrm{MeV} ; 2$, $\mathcal{E}=3.0 \mathrm{MeV} ; 3, \mathcal{E}=2.5 \mathrm{MeV} ; 4, \mathcal{E}=1.5 \mathrm{MeV}$. 


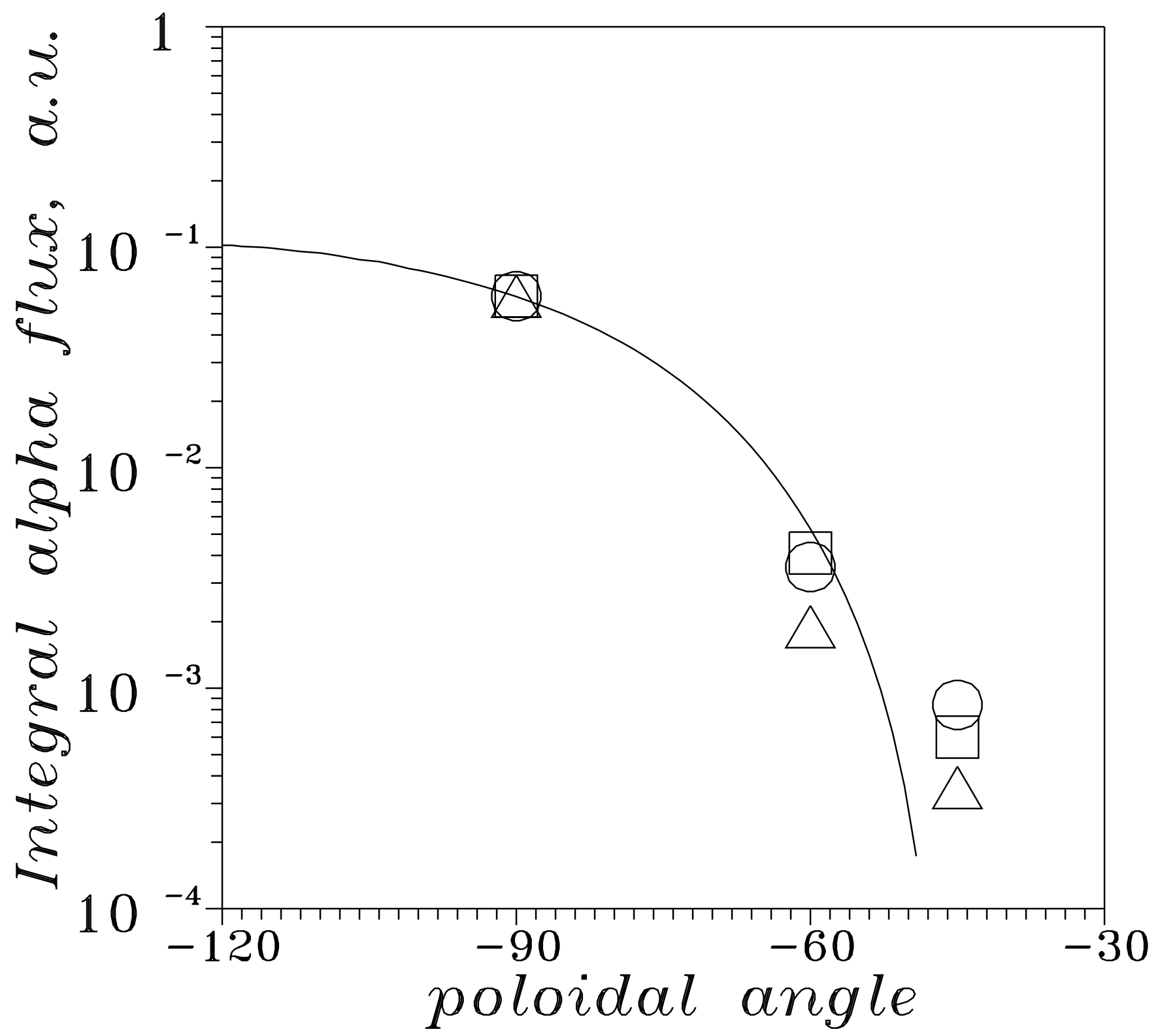

FIG. 15. The poloidal distribution of the integral alpha flux to the wall in the TFTR shot \#87530 [27,33]. Solid line, results of calculations; symbols, experimental points for three crashes. 


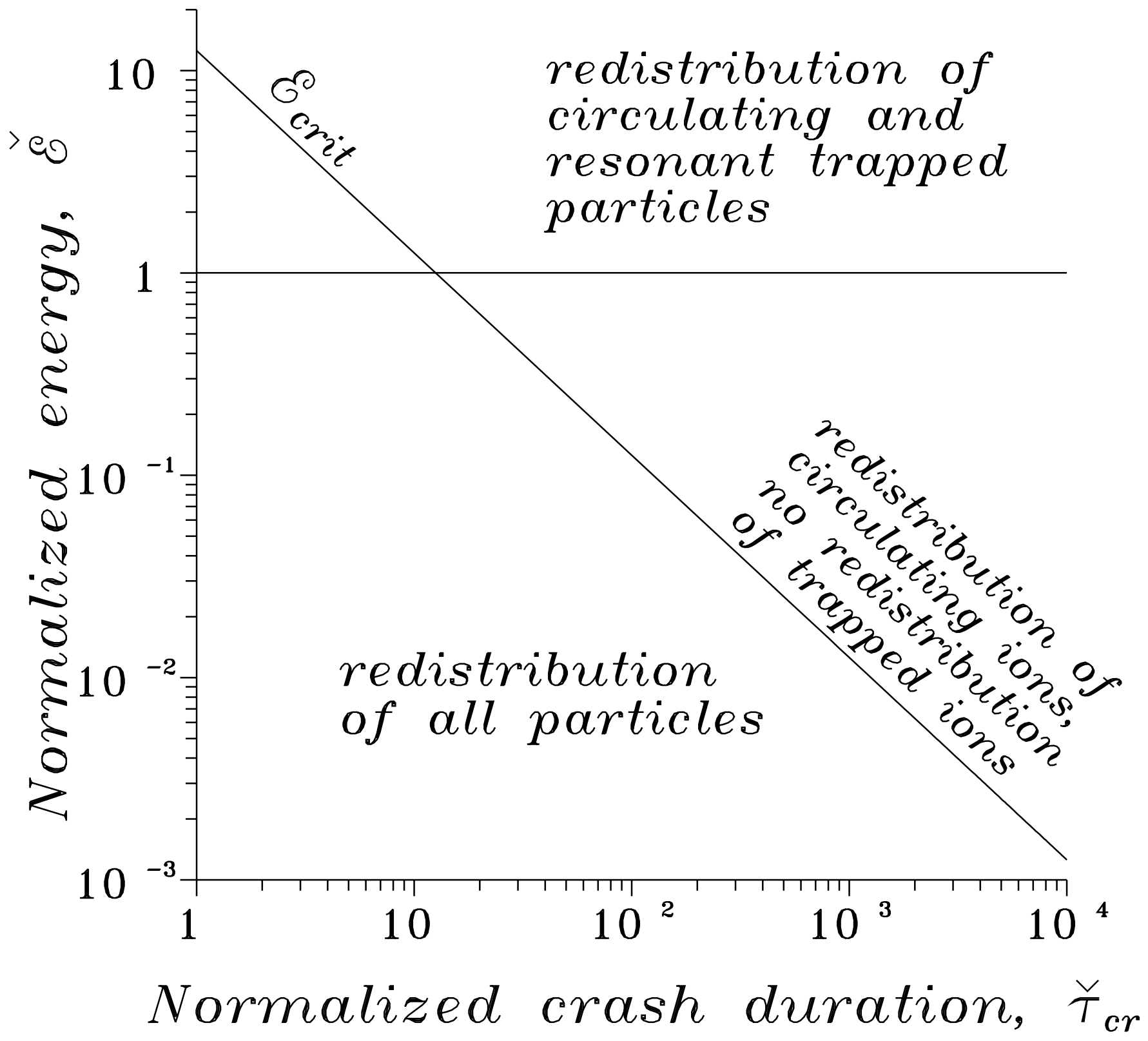

FIG. 16. Effects of sawteeth on different groups of ions depending on the normalized ion energy $\breve{\mathcal{E}}$ and the normalized crash duration $\check{\tau}_{c r}$. Notations: $\check{\mathcal{E}}=\rho^{2} /\left(\epsilon_{s} r_{s}^{2}\right), \check{\tau}_{c r}=\epsilon_{s}^{2} \omega_{B} \tau_{c r}, \rho$ is the Larmor radius of a fast ion, subscript ' $s$ ' labels values at the $q=1$ surface radius. 